\title{
Aerial Intelligent Reflecting Surface Enabled Terahertz Covert Communications in Beyond-5G Internet of Things
}

This paper was downloaded from TechRxiv (https://www.techrxiv.org).

\section{LICENSE}

CC BY 4.0

SUBMISSION DATE / POSTED DATE

23-11-2021 / 29-11-2021

\section{CITATION}

Tatar Mamaghani, Milad; Hong, Yi (2021): Aerial Intelligent Reflecting Surface Enabled Terahertz Covert Communications in Beyond-5G Internet of Things. TechRxiv. Preprint.

https://doi.org/10.36227/techrxiv.17066753.v1

$\mathrm{DOI}$

10.36227/techrxiv.17066753.v1 


\title{
Aerial Intelligent Reflecting Surface Enabled Terahertz Covert Communications in Beyond-5G Internet of Things
}

\author{
Milad Tatar Mamaghani ${ }^{\circledR}$, Graduate Student Member, IEEE, Yi Hong ${ }^{\circledR}$, Senior Member, IEEE
}

\begin{abstract}
Unmanned aerial vehicles (UAVs) are envisioned to be extensively employed for assisting wireless communications in Internet of Things (IoT). On the other hand, terahertz (THz) enabled intelligent reflecting surface (IRS) is expected to be one of the core enabling technologies for forthcoming beyond5G wireless communications that promise a broad range of data-demand applications. In this paper, we propose a UAVmounted IRS (UIRS) communication system over THz bands for confidential data dissemination from an access point (AP) towards multiple ground user equipments (UEs) in IoT networks. Specifically, the AP intends to send data to the scheduled UE, while unscheduled UEs may behave as potential adversaries. To protect information messages and the privacy of the scheduled UE, we aim to devise an energy-efficient multi-UAV covert communication scheme, where the UIRS is for reliable data transmissions, and an extra UAV is utilized as a cooperative jammer generating artificial noise (AN) to degrade unscheduled UEs detection, improving communication covertness. This poses a novel max-min optimization problem in terms of minimum average energy efficiency (mAEE), targetting to improve covert throughput and reduce UAVs' propulsion energy consumption subject to some practical constraints such as covertness which is determined analytically. Since the optimization problem is nonconvex, we tackle it via the block successive convex approximation (BSCA) approach to iteratively solve a sequence of approximated convex sub-problems, designing the binary user scheduling, AP's power allocation, maximum AN jamming power, IRS beamforming, and both UAVs' trajectory and velocity planning. Finally, we present a low-complex overall algorithm for system performance enhancement with complexity and convergence analysis. Numerical results are provided to verify our analysis and demonstrate significant outperformance of our design over other existing benchmark schemes.
\end{abstract}

Index Terms-Beyond-5G IoT networks, THz covert communications, aerial intelligent reflecting surface (AIRS), cooperative UAVs, trajectory design, resource allocation, convex optimization.

\section{INTRODUCTION}

$\mathrm{T}$ HE wireless communication and networking architectures have been witnessed revolutionary progress over the past few years. Indeed, yesteryear's smartphone-centered networks have gradually enlarged to harmoniously integrate a heterogeneous combination of massive wireless-enabled device equipment ranging from smartphones, connected intelligent

Milad Tatar Mamaghani and Yi Hong are with the Department of Electrical and Computer Systems Engineering, Faculty of Engineering, Monash University, Melbourne, VIC 3800, Australia (corresponding author e-mail: milad.tatarmamaghani@monash.edu). This work is supported by the Australian Research Council (ARC) through the ARC discovery project DP210100412. vehicles, and wearables, aiming at eventually realizing the truly connected Internet of Things (IoT) systems in the form of Internet of Everything [1].

This unprecedented proliferation of IoT devices will unquestionably drive exponential growth of wireless network traffic inasmuch as the next generation wireless systems need to offer not only larger system capacity with ultra-reliable and low latency communication but more flexible networking capabilities to adaptively cater the requirements of the IoT's dynamics [2]. In particular, reliable data dissemination is one of the core pillars of IoT-connected society, wherein the ground wireless access point (AP) typically needs to efficiently transmit information messages to IoT node(s) via multi-hop routing [3]. Such static networking architecture may admit various shortcomings [4]. First, from the perspective of capital and operational expenditures, deploying and operating traditional terrestrial infrastructures in some areas such as mountainous terrain and marine regions can be costly and inefficient. Additionally, the higher the number of intermediate nodes in multi-hop routing architectures, the more the network delay; thereby, they might be inappropriate for delay-sensitive IoT applications. Balancing the energy consumption of IoT devices is also imperative for prolonging the lifetime of IoT systems, particularly for IoT nodes having various distances to the AP. Indeed, the closer the IoT node to the AP, the quicker the energy depletion it may face due to heavier load bringing on an energy hole in the overall system. Last but not least, significant computation, synchronization, and control signaling are required for safeguarding such energy-hungry multi-hop IoT systems as they may be vulnerable to various adversary attacks due to the openness of wireless environments. To cope with the aforementioned challenges of IoT development, some promising solutions have recently been proposed in [5], [6].

\section{A UAV-IoT Communications}

Unmanned aerial vehicles (UAVs) have recently been identified as a promising technology for a myriad of civilian applications, so much so that the global market for the commercial UAV industry has been visioned to skyrocket some USD 55 billion by 2027 [7]. With on-demand swift deployment, low-cost operation and maintenance, flexible and controllable maneuverability, UAVs can be widely utilized in a broad range of scenarios such as goods shipment ${ }^{1}$, real-time

\footnotetext{
${ }^{1}$ Note that in the wake of the recent COVID-19 pandemic, some countries have practically deployed UAVs for lab sample and medical supplies delivery purposes to reduce transportation times and exposure rate [8].
} 
road traffic monitoring, precision agriculture, remote sensing, communication relaying, and wireless coverage [9]. Especially in the UAV-aided wireless communication paradigm, thanks to their flexible mobility, UAVs can establish strong line-ofsight (LoS) air-ground (AG) links towards the ground IoT devices, offering excellent wireless coverage and reduced energy consumption for resource-constraint IoT devices, and thus, overcoming the drawbacks of traditional fixed infrastructures. To this end, proper path planning/deployment and resource management for the UAV-IoT networks are of significant importance to the extent that a great deal of research has been devoted to the design of such systems (see [2], [10][14] and references therein). However, the majority of previous research efforts considered utilizing the microwave spectrum bands of sub- $6 \mathrm{GHz}$ for UAV communications, which has already been heavily occupied by traditional wireless systems leading to the spectrum crunch crisis [15]. Therefore, UAVcommunication system designs on other frequency bands, such as the promising $\mathrm{THz}$ bands for B5G UAV-IoT networks, are in demand.

\section{B THz and IRS Technologies}

Terahertz $(\mathrm{THz})$ communication is celebrated to be one of the emerging technologies for $\mathrm{B} 5 \mathrm{G}$ wireless communications thanks to the abundance of unexplored available spectrum $(0.1-10 \mathrm{THz})$ and the potentiality of fulfilling remarkable wireless capacity enhancement [15]-[18]. Recently, THz transmissions have been investigated for UAV communication applications [19], [20]. THz signals can pave the way for sharper directionality and may guarantee improved secure and reliable transmissions compared to traditional low radio frequency (RF) counterparts. However, such benefits come at the cost of some channel peculiarities, such as substantial path loss arising from distance-related attenuation as well as the water-vapor absorption phenomenon.

To compensate for their higher propagation attenuation and achieve sustainable capacity enhancement, utilizing intelligent reflecting surface (IRS) [21]-[23] has emerged as a possible solution for removing the barriers of relatively unreliable and costly conventional $\mathrm{THz}$ transmissions. Indeed, an IRS is a thin planar meta-surface composed of a large number of reconfigurable scatterers, each of which can independently collect the impinging RF signal, adjust its electromagnetic (EM) properties (e.g., the amplitude and phase shift) under the control of a smart IRS controller, and then reflect it so as to obtain the desired realization. Being low profile and lightweight, an IRS can be integrated with aerial platforms such as UAVs to enable intelligent reflection in the sky [24]. Plus, IRS can improve the spectral efficiency compared to the traditional half-duplex relaying [13], [25], [26] and also does not incur additional cost for sophisticated selfinterference cancellation algorithms conventionally utilized for full-duplex relaying [27]. An IRS passively beamforms signals without the need for any RF transceiver chains and accordingly offers the appealing feature of free of noise-corruption fullduplex relaying. Thus, several works have recently explored the benefits of deploying IRS from different perspectives. For example, $\mathrm{Wu}$ and Zhang studied the joint passive, and active beamforming design in an IRS-assisted MIMO system [28]. Pan et al. investigated the sum-rate maximization problem for an IRS-aided $\mathrm{THz}$ communication system including multiple users each demand for a different rate, via jointly designing the IRS location and phase shift, sub-band allocation, and power control [29]. Deshpande et al. considered an energy-efficient design for terrestrial IRS-assisted UAV communications via both joint trajectory, transmission power, and the phase shift optimization of an IRS with a fixed location in [30].

\section{Secure and Covert Communications}

UAV communications are appealing in terms of capacity and coverage improvement thanks to the possibility of highly LoS air-ground (AG) links. Nonetheless, the open nature of such links exposes the security of UAV-aided wireless communications, which is of pivotal importance, at significant risk. This has recently gravitated the research community to incorporate the security paradigm for designing UAV communication systems by mainly utilizing the physical layer techniques to avoid extra signaling and overheads incurred by conventional upperlayer cryptography. The information-theoretic secrecy (ITS) for safeguarding UAV communications has been extensively studied by joint design of trajectory and resource allocation in the literature [4], [13], [31]-[35]. However, preventing the content of information message from being deciphered by an eavesdropper for which the ITS aims might be inadequate when the privacy protection matters. As such, in some scenarios, the existence of legitimate transmissions needs to be sheltered from a possible vigilant adversary (or the socalled warden), and communicating terminals may desire to transfer messages covertly, since the expose of legitimate transmissions may attract the warden's attention for launching possible hostile attacks [36]-[38].

Covert communications have drawn significant interest in recent few years, where researchers have presented the square law limit, i.e., $\mathcal{O}(\sqrt{n})$ bits per $n$ channel uses can be covertly conveyed over the noisy channel between an intended sourcedestination pair without being detected by an adversary [39], [40]. In [41], the authors investigated a static IRS-assisted covert communication system and optimized the achievable rate for covert transmission. The problem of delay-sensitive covert communications over noisy channels with a finite block length has been explored in [42]. Covert communications for UAV-aided data acquisition from multiple ground users were investigated by Zhou et al. in terms of improving max-min average covert transmission rate [43]. Overall, the research endeavors about covert communication for dynamic UAV-aided systems are still in the stage of infancy [44], leaving many opportunities for future developments in various practical scenarios.

\section{$D$ Our Contributions}

In this work, we consider a wireless communication system over $\mathrm{THz}$ bands, where an AP intends to communicate with multiple ground UEs in the presence of environmental blockages. We assume that the AP sends confidential data to 
the scheduled UE (Bob) per time slot via a UAV-mounted intelligent reflecting surface (UIRS) due to no direct path inbetween, while other unscheduled UEs (Willies) may not be trustworthy. We devise a novel covert communication protocol to guarantee secure data transmission, and our detailed contributions are summarized below.

- We devise an energy-efficient multi-UAV secure covert communication scheme to protect information messages and the privacy of the scheduled UE. In particular, we employ one UIRS operating at $\mathrm{THz}$ bands for reliable data transmissions from an AP to the scheduled UE Bob per time slot, while an extra UAV is employed as a cooperative aerial jammer to generate artificial noise, aiming at degrading the detection performance of unscheduled UEs Willies.

- We obtain exclusive expressions for the minimum detection performance of unscheduled UEs in terms of the missed detection rate and the false alarm rate as the most critical metrics for covertness evaluation. A tight lower bound on the average covert data rate from AP to Bob is also derived.

- To improve covert communication while reducing network power usage, we formulate the optimization problem in terms of a new measure: the minimum average energy efficiency (mAEE). Here the mAEE is defined as the minimum average ratio between lower-bound covert throughput from AP to Bob, and UAVs' propulsion power consumption. However, the optimization problem is nonconvex and challenging to solve optimally.

- To handle this nonconvex problem, we propose a computationally efficient algorithm by applying a block coordinated successive convex approximation (BSCA) to iteratively solve a sequence of approximated convex subproblems such as user scheduling, network power allocation, IRS beamforming optimization, and joint UIRS and UCJ's trajectory and velocity optimization. We then propose a low complex overall algorithm for the system performance improvement with complexity and convergence analysis.

The rest of the paper is organized as follows. Section II introduces multi-UAV covert communication system and its setting, describing covertness requirement and formulating the energy efficient design in terms of an optimization problem. In Section III, we present an efficient iterative low-compelxity algorithm to solve the optimization problem, followed by numerical results and discussions in Section IV. Finally, we draw conclusions in Section V.

Notations: Throughout this paper, superscripts $(\cdot)^{T}$ and $(.)^{\dagger}$ denote transpose and Hermitian transpose operations. The operator $\mathbb{E}$ represents expectation, and $\|\cdot\|$ represents the Frobenius norm. Also, $\mathcal{C N}\left(0, \sigma^{2}\right)$ expresses the complex Gaussian distribution with zero mean and variance $\sigma^{2}$. The bold lower-case and upper-case letters denote a vector and matrix, respectively; the upper-case calligraphy letter indicates a set. Define $\mathbb{R}^{+}$and $\mathbb{C}$ as the sets of nonnegative real and complex numbers, respectively; $\mathbb{S}^{+}$as the set of positive semidefinite (PSD) matrices.

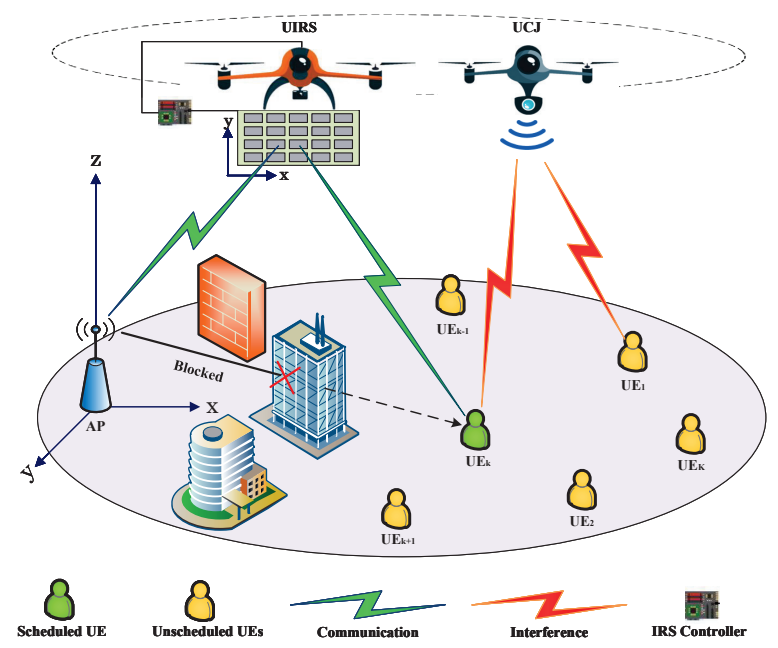

Fig. 1: System model of cooperative UIRS-UCJ aided THz covert communications in B5G-IoT networks for secure data dissemination.

\section{Multi-UAV COVERT COMMUnicATION System MODEL AND PROBLEM FORMULATION}

\section{A Multi-UAV Covert Communication System}

We consider a THz-supported wireless communication system as illustrated in Fig. 1, where a UIRS acts as a passive mobile relay to facilitate reliable end-to-end transmissions from an AP towards multiple terrestrial UEs. There is assumed to exist no direct link between AP and UEs due to severe blockage [6]. However, UIRS is likely to have strong LoS links with ground terminals due to relatively higher altitude, and mobility [45]. We assume that only one UE (Bob) is scheduled at each time instant $t$, and AP intends to covertly transmit confidential information to Bob to keep the transmission hidden from the unscheduled UEs (Willies). Such a Bob-Willies scenario may arise in large-scale distributed IoT networks where it is difficult to guarantee perfect trustworthiness and transparency of all UEs; thereby, AP needs to adapt the communication protocol according to not only the legitimate UE's requirements, but the existence of potential adversaries, e.g., Willies, whom the network operator can identify. In order to assist covert communication, a UCJ is also employed for strategically generating AN, which has been found an effective method to combat warden Willies (see [36] and references therein).

\section{B System Setting Assumptions}

We assume that AP and UCJ are equipped with a single transmit antenna, while all the terrestrial UEs are equipped with a receive antenna for data collection. Without loss of generality, we consider a 3D Cartesian coordinate system to indicate the location information of each transceiver. We denote AP's location as $\mathbf{q}_{a}=\left[x_{a}, y_{a}, 0\right]^{\mathrm{T}}$. We assume there are $K$ randomly distributed terrestrial UEs in the geographical region of interest with the fixed coordinates $\mathbf{q}_{k}=\left[x_{k}, y_{k}, 0\right]^{\mathrm{T}}$ for $\forall k \in \mathcal{K}$, where $\mathcal{K}=\{1,2, \cdots, K\}$. Further, due to UAV's limited on-board battery resource, we assume that 
UIRS and UCJ fly at the fixed altitudes $H_{r}$ and $H_{j}$ for a finite period $T$, where the altitudes are properly chosen to avoid possible collision with environmental obstacles. This fixedaltitude operation can avoid mechanical energy consumption caused by UAVs' rise and fall [11], [20].

To facilitate the UAVs' trajectory and velocity design, we adopt the time-slotted approach such that the flight duration $T$ is equally discretized into $N$ sufficiently small time slots $\delta_{t} \triangleq \frac{T}{N}$, where $\delta_{t}$ should be selected properly to balance between computational complexity and approximation accuracy. Therefore, the UIRS and UCJ's continuous trajectory and velocity sets, denoted as $\left\{\mathbf{q}_{r}(t), \mathbf{v}_{r}(t) \triangleq \frac{d \mathbf{q}_{r}(t)}{d t}\right\}$ and $\left\{\mathbf{q}_{j}(t), \mathbf{v}_{j}(t) \triangleq \frac{d \mathbf{q}_{j}(t)}{d t}\right\}$, for $0 \leq t \leq T$, can be discreted by replacing $t$ by $n \delta_{t}$, yielding the discretized sets as $\left\{\mathbf{q}_{r}[n]=\left[x_{r}[n], y_{r}[n], H_{r}\right]^{\mathrm{T}}, \mathbf{v}_{r}[n]\right\}$ and $\left\{\mathbf{q}_{j}[n]=\right.$ $\left.\left[x_{j}[n], y_{j}[n], H_{j}\right]^{\mathrm{T}}, \mathbf{v}_{j}[n]\right\}$, respectively. Moreover, we assume that each UE solely knows the channel distribution information (CDI) between itself and other UEs, while being aware of the channel between itself and the UAVs. In addition, the location information of all UEs is known to the UAVs, since all the UEs are part of the legitimate network serviced by the UAVs in different time slots.

\section{UAVs' Flight Power Model and Mission Requirements}

We consider network power consumption is dominated by the mechanical power consumption of the energy-limited rotary-wing UIRS and UCJ in terms of propulsion for aerial operation, which can be mathematically expressed as [20]

$$
\begin{aligned}
& P_{f, r}[n]=P_{o}\left(1+c_{0}\left\|\mathbf{v}_{r}[n]\right\|^{2}\right)+c_{1}\left\|\mathbf{v}_{r}[n]\right\|^{3} \\
& +P_{i}\left(\sqrt{1+c_{2}^{2}\left\|\mathbf{v}_{r}[n]\right\|^{4}}-c_{2} \mathbf{v}_{r}[n] \|^{2}\right)^{\frac{1}{2}}, \forall n \in \mathcal{N}
\end{aligned}
$$

and

$$
\begin{aligned}
& P_{f, j}[n]=P_{o}\left(1+c_{0}\left\|\mathbf{v}_{j}[n]\right\|^{2}\right)+c_{1}\left\|\mathbf{v}_{j}[n]\right\|^{3} \\
& +P_{i}\left(\sqrt{1+c_{2}^{2}\left\|\mathbf{v}_{j}[n]\right\|^{4}}-c_{2} \mathbf{v}_{j}[n] \|^{2}\right)^{\frac{1}{2}}, \forall n \in \mathcal{N}
\end{aligned}
$$

wherein $\left\{\mathbf{v}_{r}[n], \mathbf{v}_{j}[n], \forall n\right\}$ are UAVs' instantaneous velocity in time slot $n, P_{o}$ and $P_{i}$ are the UAVs' blade profile power and induced power in hovering mode, respectively, and $c_{0}$, $c_{1}$, and $c_{2}$ are some mechanical and environmental-related constants [46]. When a UAV is aloft, i.e., $\left\|\mathbf{v}_{r(j)}[n]\right\|=0$, its mechanical power consumption is $\bar{P}_{f}=P_{i}+P_{o}$ which is not necessarily the minimum power consumption and thereby hovering at a specific point may not be an energy efficient approach for UAV deployment.

Here, we consider that UAVs are deployed to periodically fly over the sky providing covert communications to the UEs; thereby, flight constraints to the UIRS can be imposed by

$$
\begin{array}{ll}
\mathrm{C} 1: & \mathbf{q}_{r}[1]=\mathbf{q}_{r}[N]=\mathbf{q}_{r}^{I}, \\
& \left\|\mathbf{q}_{r}[n]-\mathbf{q}_{a}\right\| \leq \sqrt{R_{p}^{2}+H_{r}^{2}}, \forall n \in \mathcal{N} \\
& \mathbf{q}_{r}[n+1]=\mathbf{q}_{r}[n]+\mathbf{v}_{r}[n] \delta_{t}, \forall n \in \mathcal{N} \backslash\{N\} \\
& \left\|\mathbf{v}_{r}[n]\right\| \leq v_{r}^{\max }, \forall n \in \mathcal{N} \\
& \left\|\mathbf{v}_{r}[n+1]-\mathbf{v}_{r}[n]\right\| \leq a_{r}^{\max }, \forall n \in \mathcal{N} \backslash\{N\}
\end{array}
$$

where (3a) is to ensure a periodic flight on the grounds that the UIRS has to return to the initial location by the end of the last time slot, (3b) restricts UIRS's flying region within the permitted zone, (3c), (3d), and (3e) represent UIRS's mobility constraints from practical perspective. Similarly, UCJ's flight constraints can be given by

$$
\begin{array}{ll}
\mathrm{C} 2: & \mathbf{q}_{j}[1]=\mathbf{q}_{j}[N]=\mathbf{q}_{j}^{I}, \\
& \left\|\mathbf{q}_{j}[n]-\mathbf{q}_{a}\right\| \leq \sqrt{R_{p}^{2}+H_{j}^{2}}, \forall n \in \mathcal{N} \\
& \mathbf{q}_{j}[n+1]=\mathbf{q}_{j}[n]+\mathbf{v}_{j}[n] \delta_{t}, \forall n \in \mathcal{N} \backslash\{N\} \\
& \left\|\mathbf{v}_{j}[n]\right\| \leq v_{j}^{\max }, \forall n \in \mathcal{N} \\
& \left\|\mathbf{v}_{j}[n+1]-\mathbf{v}_{j}[n]\right\| \leq a_{j}^{\max }, \forall n \in \mathcal{N} \backslash\{N\}
\end{array}
$$

where $\mathbf{q}_{r}^{I}$ and $\mathbf{q}_{j}^{I}$ are UAVs' predetermined stations per flight, $\left\{v_{r}^{\max }, v_{j}^{\max }\right\}$ and $\left\{a_{r}^{\max }, a_{j}^{\max }\right\}$ are the UAVs' instantaneous maximum speeds and accelerations, respectively. To avoid possible collision in the multi-UAV system, we need to consider safety distance between the UAVs, represented as

$$
\text { C3 : } \quad\left\|\mathbf{q}_{r}[n]-\mathbf{q}_{j}[n]\right\| \geq D_{s}, \forall n \in \mathcal{N}
$$

where $D_{s}$ denotes the minimum required distance between the two UAVs throughout the periodic mission.

\section{Transmission Strategy}

We assume that direct links between AP and UEs are absent due to severe blockage or considerable distance, necessitating the significance of aerial platforms such as UIRS to establish a reliable wireless link for data dissemination. To support UIRSassisted downlink covert data transmission service to multiple UEs, we employ the time division multiple access (TDMA) protocol, wherein at most one UE is scheduled per time slot for intended data transmission, while the other unscheduled UEs play as adversaries attempting to detect the presence of communication. By letting $\alpha_{k}[n]$ be a binary user scheduling variable for UE $k$ in time slot $n$, we have the user scheduling constraint as

$$
\begin{gathered}
\mathrm{C} 4: \quad \alpha_{k}[n] \in\{0,1\}, \forall k \in \mathcal{K}, \forall n \in \mathcal{N} \\
\sum_{k=1}^{K} \alpha_{k}[n] \leq 1, \forall n \in \mathcal{N}
\end{gathered}
$$

where $\alpha_{k}[n]=1$ if UE $k$ is scheduled in time slot $n$, and zero otherwise.

\section{E Channel Modeling}

Since our work examines transmissions over $\mathrm{THz}$ frequencies, the THz channel gain for direct links from UCJ to UE $k$ in time slot $n$ can be denoted, similar in [19], [20], [47], as

$$
\begin{gathered}
h_{j k}[n]=\left(\frac{C}{4 \pi f_{c}\left\|\mathbf{q}_{j}[n]-\mathbf{q}_{k}\right\|^{\frac{\rho}{2}}}\right) \exp \left(\frac{-j 2 \pi\left\|\mathbf{q}_{j}[n]-\mathbf{q}_{k}\right\|}{\lambda_{c}}\right) \\
\quad \times \exp \left(-\frac{\kappa\left(f_{c}, \mu\right)\left\|\mathbf{q}_{j}[n]-\mathbf{q}_{k}\right\|}{2}\right), \forall k \in \mathcal{K}, n \in \mathcal{N}
\end{gathered}
$$

wherein $\lambda_{c} \triangleq \frac{C}{f_{c}}$ is the transmission wavelength in meter, $C \approx 3 \times 10^{8} \mathrm{~m} \mathrm{~s}^{-1}$ is the speed of light, and $f_{c}$ specifies the 


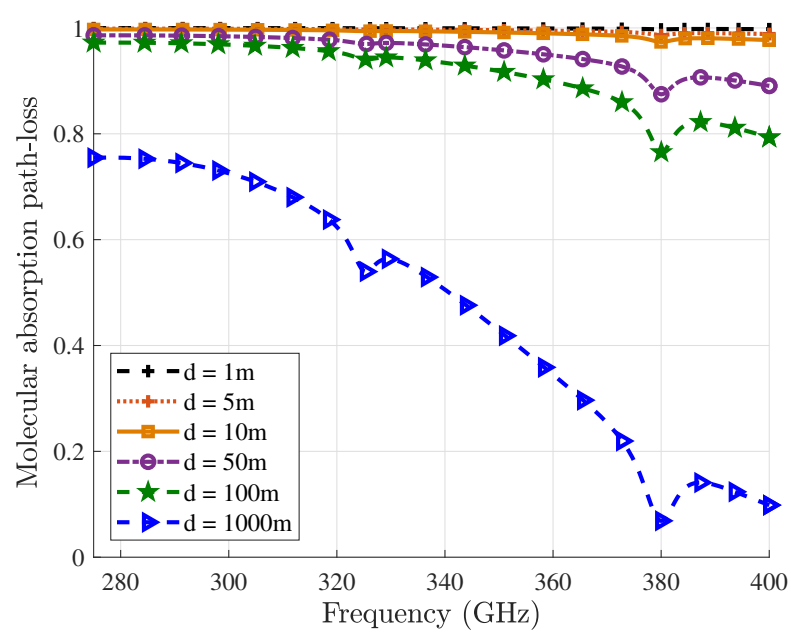

Fig. 2: Illustration of how THz molecular absorption path-loss varies with carrier frequency and distance $(d)$ for standard atmospheric condition.

carrier frequency. Furthermore, $\kappa\left(f_{c}, \mu\right)$ represents the overall molecular absorption coefficient of $\mathrm{THz}$ channels as a function of $f_{c}$ and the volume of the mixing ratio of water vapor $\mu$, describing the relative absorbing area of the molecules in the wireless medium per unit volume. It should be stressed that the main cause of absorption loss in $\mathrm{THz}$ frequency ranges is the water vapor molecules that cause a discrete but deterministic loss to the signals in the frequency domain [18], [47].

Remark 1. The molecular absorption loss in $275-400 \mathrm{GHz}$ can be modelled, according to [47], as $L_{a}^{-1}\left(f_{c}, d, \mu\right)=$ $\exp \left(-\kappa\left(f_{c}, \mu\right) d\right)$ where $d$ is the distance, and

$$
\begin{aligned}
& \kappa\left(f_{c}, \mu\right)=\frac{0.2205 \mu(0.133 \mu+0.0294)}{(0.4093 \mu+0.0925)^{2}+\left(\frac{f_{c}}{100 C}-10.835\right)^{2}} \\
& +\frac{2.014 \mu(0.1702 \mu+0.0303)}{(0.537 \mu+0.0956)^{2}+\left(\frac{f_{c}}{100 C}-12.664\right)^{2}}+5.54 \times 10^{-37} f_{c}^{3} \\
& -3.94 \times 10^{-25} f_{c}^{2}+9.06 \times 10^{-14} f_{c}-6.36 \times 10^{-3},
\end{aligned}
$$

in which $\mu$ can be evaluated as

$$
\mu=6.1121\left(3.46 \times 10^{-8} P+1.0007\right) \frac{\phi}{P} \exp \left(\frac{17.502 T}{240.97+T}\right)
$$

wherein $\phi$ stands for the relative humidity in percentage, $P$ denotes the pressure in $\mathrm{Pa}$, and $T$ is measured in ${ }^{\circ} \mathrm{C}$. The $\mathrm{THz}$ link molecular absorption path-loss model given above and illustrated in Fig. 2 was shown to have high accuracy for up to $1 \mathrm{~km}$ links in standard atmospheric conditions, i.e., the temperature of $296 \mathrm{~K}$ and pressure of $101325 \mathrm{~Pa}$. However, it is worth mentioning that nonstandard atmospheric conditions can also be described via this model.

On the other hand, the cascaded channel gain of the APUIRS-UE $k \in \mathcal{K}$ in time slot $n \in \mathcal{N}$ can be represented, in the far field scenario, as [48]

$$
\begin{aligned}
& \tilde{h}_{a r k}[n]=\left(\frac{C}{8 \pi \sqrt{\pi} f_{c}\left\|\mathbf{q}_{r}[n]-\mathbf{q}_{a}\right\|^{\frac{\rho}{2}}\left\|\mathbf{q}_{r}[n]-\mathbf{q}_{k}\right\|^{\frac{\rho}{2}}}\right) \\
& \times \exp \left(-\frac{j 2 \pi\left(\left\|\mathbf{q}_{r}[n]-\mathbf{q}_{a}\right\|+\left\|\mathbf{q}_{r}[n]-\mathbf{q}_{k}\right\|\right)}{\lambda_{c}}\right) \\
& \times \exp \left(-\frac{\kappa\left(\left\|\mathbf{q}_{r}[n]-\mathbf{q}_{a}\right\|+\left\|\mathbf{q}_{r}[n]-\mathbf{q}_{k}\right\|\right)}{2}\right),
\end{aligned}
$$

where $\kappa \triangleq \kappa\left(f_{c}, \mu\right)$ for notation simplicity. Consider there is an IRS as a uniform planar array (UPA) deployed at UIRS parallel to the ground. Let $L_{x}$ and $L_{y}$ be the number of reflecting elements alongside the $\mathrm{x}$ and $\mathrm{y}$-axes of the IRS (see Fig. 1), respectively, so the total number of reflecting elements is $L=L_{x} L_{y}$. Here, we assume that the $3 \mathrm{D}$ coordinate of the first element of the IRS (the IRS element shown at the origin in Fig. 1) equals to the instantaneous location of UIRS, i.e., $\mathbf{q}_{r}[n]$. Therefore, the transmission vector from AP towards the first element of the IRS is $\left(\mathbf{q}_{r}[n]-\mathbf{q}_{a}\right)$, and also, the difference vector from the IRS can be represented as $\Delta \mathbf{r}_{l_{x}, l_{y}}=\left[\left(l_{x}-1\right) \delta_{x},\left(l_{y}-1\right) \delta_{y}, 0\right]^{\mathrm{T}}$, where $l_{x}$ and $l_{y}$ represent the $l_{x}$-th row and $l_{y}$-th column of the IRS, $\delta_{x}$ and $\delta_{y}$ denote the element separation alongside $\mathrm{x}$ and $\mathrm{y}$ axes. Accordingly, the relative phase difference between the signal received by the first element and the $\left(l_{x}, l_{y}\right)$-th element of the IRS in time slot $n$ can be represented by

$$
\theta_{l}[n]=\frac{2 \pi\left(\mathbf{q}_{r}[n]-\mathbf{q}_{a}\right)^{\mathrm{T}} \Delta r_{l_{x}, l_{y}}}{\lambda_{c}\left\|\mathbf{q}_{r}[n]-\mathbf{q}_{a}\right\|}, \forall n \in \mathcal{N}
$$

where the subscript $l=\left(l_{x}-1\right) \times L_{y}+l_{y}$. Henceforth, the received array vector from AP to UIRS is given by

$$
\mathbf{e}_{a}[n]=\left[\mathrm{e}^{-j \theta_{l}[n]}, \cdots, \mathrm{e}^{-j \theta_{L}[n]}\right]^{\mathrm{T}}, \forall n \in \mathcal{N}
$$

Similarly, the relative phase difference between the first and $\left(l_{x}, l_{y}\right)$-th element of the IRS's reflected beams towards UE $k$ is given by

$$
\beta_{l}^{k}[n]=\frac{2 \pi\left(\mathbf{q}_{k}-\mathbf{q}_{r}[n]\right)^{\mathrm{T}} \Delta r_{l_{x}, l_{y}}}{\lambda_{c}\left\|\mathbf{q}_{k}-\mathbf{q}_{r}[n]\right\|}, \forall k \in \mathcal{K}, n \in \mathcal{N}
$$

Therefore, the transmit array beam from UIRS to the $k$-th UE in time slot $n$ can be represented as

$$
\mathbf{e}_{k}[n]=\left[\mathrm{e}^{-j \beta_{1}^{k}[n]}, \cdots, \mathrm{e}^{-j \beta_{L}^{k}[n]}\right]^{\mathrm{T}}, \forall k \in \mathcal{K}, n \in \mathcal{N}
$$

Hence, we can express the channel gain of AP-UIRS-UE $k$ as

$$
h_{\text {ark }}[n]=\mathbf{e}_{k}^{\dagger}[n] \Phi[n] \mathbf{e}_{a}[n] \tilde{h}_{\text {ark }}[n], \forall k \in \mathcal{K}, n \in \mathcal{N}
$$

where $\Phi[n]$ is an $L$-by- $L$ beamforming matrix of the IRS defined as

$$
\Phi[n] \triangleq \operatorname{diag}\left(\rho_{1}[n] \mathrm{e}^{j \phi_{1}[n]}, \cdots, \rho_{L}[n] \mathrm{e}^{j \phi_{L}[n]}\right)
$$

each element of its main diagonal represents both the amplitude and the phase shift of the $\left(l_{x}, l_{y}\right)$-th element of the IRS in time slot $n$ with the constraints given by

$$
\begin{aligned}
\mathrm{C} 5: \quad & 0 \leq \rho_{l}[n] \leq 1, \quad \forall l \in \mathcal{L}, n \in \mathcal{N} \\
& 0 \leq \phi_{l}[n] \leq 2 \pi, \quad \forall l \in \mathcal{L}, n \in \mathcal{N}
\end{aligned}
$$

where $\mathcal{L}=\{1,2, \cdots, L\}$. 


\section{F Signals Representation}

In the $n$-th time slot, the signal received by the $k$-th UE is given by

$$
\begin{aligned}
y_{k}[n] & =\sqrt{p_{a}[n]} h_{a r k}[n] x_{a}[n] \\
& +\sqrt{p_{j}[n]} h_{j k}[n] x_{j}[n]+\delta_{k}[n], \forall k \in \mathcal{K}, n \in \mathcal{N}
\end{aligned}
$$

where $\delta_{k}[n] \in \mathcal{C N}\left(0, \sigma^{2}\right)$ is the AWGN at UE $k, x_{a}[n]$ with normalized unit power denotes the information signal transmitted by the AP towards the scheduled UE $k, x_{j}[n]$ represents the AN transmitted by UCJ following $\mathbb{E}\left\{x_{a}^{2}[n]\right\}=1$. Further, $p_{a}[n]$ and $p_{j}[n]$ denote AP and UCJ's transmit powers in time slot $n$. In this work, we assume that $p_{j}[n]$ is a random variable with a uniform distribution in the interval $\left[0, \hat{p}_{j}[n]\right], \forall n$, where in $\hat{p}_{j}[n]$ denotes the peak AN transmission power by UCJ in time slot $n$, with the following probability density function (pdf) and cumulative distribution function (cdf) as

$$
\begin{aligned}
& f_{p_{j}[n]}(x)= \begin{cases}\frac{1}{\hat{p}_{j}[n]}, & 0 \leq x \leq \hat{p}_{j}[n] \\
0, & \text { o.w. }\end{cases} \\
& F_{p_{j}[n]}(x)= \begin{cases}0, & x \leq 0 \\
\frac{x}{\hat{p}_{j}[n]}, & 0 \leq x \leq \hat{p}_{j}[n] \\
1, & x \geq \hat{p}_{j}[n]\end{cases}
\end{aligned}
$$

with expected value $\mathbb{E}\left\{p_{j}[n]\right\}=\frac{\hat{p}_{j}[n]}{2}$.

It is worth pointing out that AP's transmit power and the peak AN power of UCJ are generally subject to maximum instantaneous and total network power budget constraints represented as

$$
\begin{aligned}
\text { C6 }: & 0 \leq p_{a}[n] \leq p_{a}^{\max }, \forall n \in \mathcal{N} \\
& 0 \leq \hat{p}_{j}[n] \leq p_{j}^{\max }, \forall n \in \mathcal{N} \\
& \sum_{n=1}^{N} p_{a}[n]+\hat{p}_{j}[n] \leq p^{\text {tot }},
\end{aligned}
$$

Remark 2. By introducing randomness in the UCJ's AN transmissions, we can create uncertainty in the received power at Willies (unscheduled UEs) to assist covert transmissions to Bob (the scheduled UE). From a conservative point of view, we assume that Willies know the distribution information of the UCJ's AN transmit powers, which is the worst-case scenario since it becomes much easier for them to make a decision less erroneous than the cases without such information.

Following (16), the average channel capacity from AP to UE $k$ taking over the randomness nature of AN transmission powers by UCJ can be obtained as

$$
\begin{aligned}
& \bar{R}_{k}[n]=\mathbb{E}_{p_{j}[n]}\left\{\mathrm{W} \log _{2}\left(1+\frac{p_{a}[n] g_{a r k}[n]}{p_{j}[n] g_{j k}+\sigma_{k}^{2}[n]}\right)\right\}, \\
& \stackrel{(a)}{\geq} \mathrm{W} \log _{2}\left(1+\frac{p_{a}[n] g_{a r k}[n]}{\frac{1}{2} \hat{p}_{j}[n] g_{j k}[n]+\sigma_{k}^{2}[n]}\right) \triangleq \bar{R}_{k}^{l b}[n],
\end{aligned}
$$

wherein $\mathrm{W}$ is the allocated transmission bandwidth in $\mathrm{Hz}$, $g_{\text {ark }} \triangleq\left\|h_{\text {ark }}[n]\right\|^{2}, g_{j k}[n] \triangleq\left\|h_{j k}[n]\right\|^{2}$. Further, $(a)$ follows from applying Jensen's inequality theorem wherein given $X$ be an arbitrary random variable then according to [49] we have

$$
\mathbb{E}\{f(X)\} \geq f(\mathbb{E}\{X\}) \Longleftrightarrow f(X) \text { is convex }
$$

As a result, owing to the convexity of the function $\log \left(1+\frac{a}{b x+1}\right)$ with respect to (w.r.t) the variable $x$ in the domain of $x \geq-\frac{2+a}{2 b}$ for $a, b \geq 0$ [27], the tight lower-bound expression $\bar{R}_{k}^{l b}[n]$ can be obtained.

\section{G Covert Communication Requirement and Analysis}

For the non-colluding Willies, i.e., each of the unscheduled UEs (a.k.a Willies) $\mathcal{W}=\mathcal{K} \backslash\{k\}$ independently attempts for conducting malicious activity in terms of signal transmission detection. Thus, each of Willies encounters a binary hypothesis testing problem to independently decide whether AP has transmitted information signal towards the scheduling UE $k$. This non-colluding scenario is valid as the UEs are randomly distributed in the region, and each potentially serves as a scheduled UE in some specific time slots during the mission. Therefore, the received signal at the $m$-th Willie in time slot $n$ can be represented as

$$
y_{m}[n]= \begin{cases}\sqrt{p_{j}[n]} h_{j m}[n] x_{j}[n]+\delta_{m}[n], & \mathcal{H}_{0} \\ \sqrt{p_{a}[n]} h_{a r m}[n] x_{a}[n] & \\ +\sqrt{p_{j}[n]} h_{j m}[n] x_{j}[n]+\delta_{m}[n], & \mathcal{H}_{1}\end{cases}
$$

where $\delta_{m}[n] \sim \mathcal{C N}\left(0, \sigma_{m}^{2}\right)$ is the AWGN at Willie $m \in \mathcal{W}$. $\mathcal{H}_{0}$ is the null hypothesis stating that AP has not transmitted information signal, whereas $\mathcal{H}_{1}$ is the alternative hypothesis. Here, we assume that Willies use a radiometer for signal detection, conducting a threshold test on the average power received. The optimal decision rule for minimizing the detection error at Willie $m$ in time slot $n$ is written as

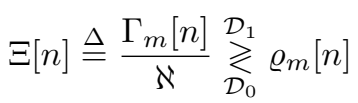

where $\aleph$ is the total number of channel uses per time slot, $\Gamma_{m}[n] \triangleq \sum_{j=1}^{\aleph}\left\|y_{m}^{(j)}[n]\right\|^{2}$ is the total received power at Willie $m$ in time slot $n$, and $\varrho_{m}[n]$ represents the corresponding detection threshold, while $\mathcal{D}_{0}$ and $\mathcal{D}_{1}$ denote the decisions in favor of hypothesis $\mathcal{H}_{0}$ and $\mathcal{H}_{1}$, respectively. Here we adopt a widely used assumption in covert communications: $\aleph \longrightarrow \infty$, implying that each Willie can observe an infinite number of samples, which is, in fact, an upper bound on the number of received samples in practice. Nonetheless, owing to the fact that the number of symbols transmitted per time slot gets increased with the communication bandwidth and thanks to the abundance of available bandwidth in $\mathrm{THz}$ frequencies, this assumption of approximately infinite channel uses per slot can be well justified. Therefore, (22) can be simplified as

$$
\Xi[n]= \begin{cases}p_{j}[n] g_{j m}+\sigma_{m}^{2}, & \mathcal{H}_{0} \\ p_{a}[n] g_{a r m}[n]+p_{j}[n] g_{j m}+\sigma_{m}^{2}, & \mathcal{H}_{1}\end{cases}
$$

False Alarm Rate: If Willie $m$ decides that AP has sent data to the scheduled UE $k$ in the $n$-th time slot, while AP has not sent any data, we have False Alarm (FA) occurrence with probability of

$$
\begin{aligned}
P_{m}^{F A}[n] & \triangleq \operatorname{Pr}\left\{\mathcal{D}_{1} \mid \mathcal{H}_{0}\right\} \\
& =\operatorname{Pr}\left\{p_{j}[n] g_{j m}[n]+\sigma_{m}^{2}[n] \geq \varrho_{m}[n]\right\},
\end{aligned}
$$


Missed Detection Rate: If Willie $m$ decides that AP has not transmitted data to the scheduled UE $k$, when AP did, then we say that a Missed Detection (MD) incident has occurred with the probability given by

$$
\begin{aligned}
& \mathrm{P}_{k, m}^{M D}[n]=\operatorname{Pr}\left\{\mathcal{D}_{0} \mid \mathcal{H}_{1}\right\} \\
& \quad=\operatorname{Pr}\left\{p_{a}[n] g_{a r m}[n]+p_{j}[n] g_{j m}[n]+\sigma_{m}^{2}[n] \leq \varrho_{m}[n]\right\},
\end{aligned}
$$

Then the detection error rate at Willie $m$ given $k$-th Bob in time slot $n$ is expressed by

$$
\zeta_{k, m}[n]=P_{m}^{F A}[n]+\mathrm{P}_{k, m}^{M D}[n], \forall n, k, m
$$

In general, Willie $m \in \mathcal{W} \triangleq \mathcal{K} \backslash\{k\}$ tries to minimize the error detection rate in (26), while the UIRS-UCJ aims at ensuring this minimum error detection, denoted by $\zeta_{m, k}^{\star}[n]$, being no more than some specific value at every Willie in time slot $n$, i.e., $\zeta_{m, k}^{\star}[n] \geq 1-\varepsilon$. It is worth mentioning that $\varepsilon$ is typically a small, non-negative constant denoting the covertness requirement for data dissemination in the system. In the following, we first derive analytical expressions for the FA and MD probabilities, based on which we then obtain optimal detection threshold from Willies' perspective.

Exploiting the distribution of random variables $p_{j}[n], \forall n \in$ $\mathcal{N}$, given by (17) and (18), we can rewrite (24) as

$$
\begin{aligned}
P_{m}^{F A}[n] & =1-F_{p_{j}[n]}\left(\frac{\varrho_{m}[n]-\sigma_{m}^{2}[n]}{g_{j m}[n]}\right) \\
& = \begin{cases}1, & \varrho_{m}[n] \leq \sigma_{m}^{2}[n] \\
1-\frac{\varrho_{m}[n]-\sigma_{m}^{2}[n]}{\hat{p}_{j}[n] g_{j m}[n]}, & \sigma_{m}^{2}[n]<\varrho_{m}[n] \leq \chi_{2} \\
0, & \varrho_{m}[n]>\chi_{2}\end{cases}
\end{aligned}
$$

wherein $\chi_{2} \triangleq \hat{p}_{j}[n] g_{j m}[n]+\sigma_{m}^{2}[n]$. Similarly, we can analytically calculate (25) as

$$
\begin{aligned}
P_{k, m}^{M D}[n] & =F_{p_{j}[n]}\left(\frac{\varrho_{m}[n]-p_{a}[n] g_{a r m}[n]-\sigma_{m}^{2}[n]}{g_{j m}[n]}\right) \\
& = \begin{cases}0, & \varrho_{m}[n] \leq \chi_{1} \\
\frac{\varrho_{m}[n]-p_{a}[n] g_{a r m}[n]-\sigma_{m}^{2}[n]}{\hat{p}_{j}[n] g_{j m}[n]}, & \chi_{1}<\varrho_{m}[n] \leq \chi_{3} \\
1, & \varrho_{m}[n]>\chi_{3}\end{cases}
\end{aligned}
$$

wherein $\chi_{1} \triangleq p_{a}[n] g_{a r m}[n]+\sigma_{m}^{2}[n]$ and $\chi_{3} \triangleq p_{a}[n] g_{a r m}[n]+$ $\hat{p}_{j}[n] g_{j m}[n]+\sigma_{m}^{2}[n]$. We note that if $\hat{p}_{j}[n] g_{j m}[n] \leq$ $p_{a}[n] g_{a r m}[n]$, then by setting $\varrho_{m}[n]=p_{a}[n] g_{a r m}[n]+\sigma_{m}^{2}[n]$, Willie $m$ can achieve zero error detection rate. Hence, we consider the non-trivial case wherein $\chi_{1} \leq \chi_{2} \leq \chi_{3}$ which is equivalent with

$$
\hat{p}_{j}[n] g_{j m}[n] \geq p_{a}[n] g_{a r m}[n], \forall n \in \mathcal{N}, m \in \mathcal{W}
$$

Hence, the following analyses are based on the aforementioned critical assumption for covertness requirement. Now, we can compute $\zeta_{k, m}[n]$ given by (26), as

$$
\zeta_{k, m}[n]= \begin{cases}1, & \varrho_{m}[n] \leq \sigma_{m}^{2}[n] \\ 1-\frac{\varrho_{m}[n]-\sigma_{m}^{2}[n]}{\hat{p}_{j}[n] g_{j m}[n]}, & \sigma_{m}^{2}[n] \leq \varrho_{m}[n]<\chi_{1} \\ 1-\frac{p_{a}[n] g_{a r m}[n]}{\hat{p}_{j}[n] g_{j m}[n]}, & \chi_{1} \leq \varrho_{m}[n]<\chi_{2} \\ \frac{\varrho_{m}[n]-p_{a}[n] g_{a r m}[n]-\sigma_{m}^{2}[n]}{\hat{p}_{j}[n] g_{j m}[n]}, & \chi_{2} \leq \varrho_{m}[n]<\chi_{3} \\ 1, & \varrho_{m}[n] \geq \chi_{3}\end{cases}
$$

It is evident that Willies will not set the decision threshold lower than noise variance $\sigma_{m}^{2}[n]$ or higher than $\chi_{3}$ to ensure that the resulting detection error probability will be less than 1 ; otherwise, the detection performance would be the same as of a random guess. Further, we can see that $\zeta_{k, m}[n]$ is a decreasing function in the range $\sigma_{m}^{2}[n] \leq \varrho_{m}[n]<\chi_{1}$ and increasing function in the range $\chi_{2} \leq \varrho_{m}[n]<\chi_{3}$ w.r.t $\varrho_{m}[n]$ and also behaves as a constant function when $\chi_{1} \leq \varrho_{m}[n]<\chi_{2}$. Therefore, considering that $\zeta_{k, m}[n]$ is a continuous function of $\varrho_{m}[n]$, the optimal decision threshold to be set by Willie $m$ should be in the range $\chi_{1} \leq \varrho_{m}[n] \leq \chi_{2}$, resulting in the minimum detection error rate

$$
\zeta_{m, k}^{\star}[n]=1-\frac{p_{a}[n] g_{a r m}[n]}{\hat{p}_{j}[n] g_{j m}[n]}, \forall n \in \mathcal{N}, k \in \mathcal{K}, m \in \mathcal{W}
$$

Then, the covert communication constraint can be stated as

$$
\mathrm{C} 7: \sum_{k=1}^{K} \alpha_{k}[n] \min _{m \in \mathcal{W}} \zeta_{m, k}^{\star}[n] \geq 1-\varepsilon, \forall n \in \mathcal{N}
$$

wherein $\zeta_{m, k}^{\star}[n]$ is in (30).

Remark 3. It is worth pointing out that, as per the minimum error detection rate obtained in (30), $\zeta_{m, k}^{\star}[n]$ decreases with the AP's transmit power as well as the downlink channel quality but increases when UCJ's maximum AN transmission power gets increased or the quality of the interference links improves. Therefore, it requires to carefully design the UAVs' trajectory and the communication resources to balance transmission quality and communication covertness.

\section{PROBlem Formulation AND PROPOSED LOW-COMPLEX SOLUTION}

To devise an energy-efficient UIRS-assisted covert communication system, we first formulate the optimization problem aiming to improve the minimum average energy efficiency (mAEE) of the network. Here the mAEE is defined as the minimum of average-ratios of lower-bound throughput given by (20) over the UAVs' propulsion power consumption ${ }^{2}$ as

$$
\begin{aligned}
& (\mathrm{P}): \underset{\boldsymbol{\alpha}, \mathbf{P}, \boldsymbol{\Phi}, \mathbf{Q}_{r}, \mathbf{Q}_{j}}{\operatorname{maximize}} \min _{k \in \mathcal{K}} \frac{1}{N} \sum_{n=1}^{N} \frac{\alpha_{k}[n] \bar{R}_{k}^{l b}[n]}{P_{f, r}[n]+P_{f, j}[n]} \\
& \text { s.t. } \quad \mathrm{C} 1-\mathrm{C} 7,
\end{aligned}
$$

Note that problem $(\mathrm{P})$ is a mixed-integer fractional nonconvex non-linear programming, which is challenging to solve

\footnotetext{
${ }^{2}$ Mechanical power consumption of the UAVs is the dominant power consumption of the network compared to the power resource required for transmissions, signal processing, circuitry, and so forth.
} 
optimally. Indeed, the major challenge in solving $(\mathrm{P})$ arises from the binary user scheduling constraint $\mathrm{C} 4$, nonconvex constraints $\mathrm{C} 3$ and $\mathrm{C} 7$, and the highly coupled optimization variables in the fractional-form objective function. To embark on the non-convexity and make the problem tractable, we propose a computationally efficient algorithm by applying a block coordinated successive convex approximation (BSCA) to iteratively solve a sequence of approximated convex subproblems by employing several techniques. Specifically, we split problem $(\mathrm{P})$ into the following sub-problems with different blocks of variables: i) user scheduling sub-problem to optimize $\boldsymbol{\alpha}$, ii) network transmission power sub-problem to optimize $\left.\mathbf{P}=\left\{\mathbf{P}_{a}, \hat{\mathbf{P}}_{j}\right\}, i i i\right)$ beamforming matrices subproblem to improve $\boldsymbol{\Phi}=\{\Phi[n], \forall n \in \mathcal{N}\}$, iv) UIRS's joint trajectory and velocity sub-problem to improve $\mathbf{Q}_{r}=$ $\left\{\mathbf{q}_{r}[n], \mathbf{v}_{r}[n], \forall n \in \mathcal{N}\right\}$ and $v$ ) UCJ's joint trajectory and velocity sub-problem to improve $\mathbf{Q}_{j}=\left\{\mathbf{q}_{j}[n], \mathbf{v}_{j}[n], \forall n \in\right.$ $\mathcal{N}\}$. Next, we solve each of them while keeping the other blocks fixed, then propose an overall low-complex algorithm to iteratively attain the approximate solution of (32).

\section{A Sub-problem I: User Scheduling Optimization}

By keeping the optimization blocks $\mathbf{P}, \boldsymbol{\Phi}, \mathbf{Q}_{r}, \mathbf{Q}_{j}$ fixed and relaxing the binary constraint (6) into a continuous constraints, we can rewrite $(\mathrm{P} 1)$ equivalently as

$$
\begin{aligned}
&(\mathrm{P} 1): \underset{\boldsymbol{\alpha}}{\operatorname{maximize}} \min _{k \in \mathcal{K}} \frac{1}{N} \sum_{n=1}^{N} A_{k, n} \alpha_{k}[n] \\
& \text { s.t. } \quad \sum_{k=1}^{K} B_{k, n} \alpha_{k}[n] \geq 1-\varepsilon, \forall n \in \mathcal{N} \\
& 0 \leq \alpha_{k}[n] \leq 1, \forall k \in \mathcal{K}, n \in \mathcal{N} \\
& \sum_{n=1}^{N} \sum_{k=1}^{K}\left(\alpha_{k}[n]-\alpha_{k}^{2}[n]\right) \leq 0 \\
& \sum_{k=1}^{K} \alpha_{k}[n] \leq 1, \forall n \in \mathcal{N}
\end{aligned}
$$

wherein

$A_{k, n}=\frac{\bar{R}_{k}^{l b}[n]}{P_{f, r}[n]+P_{f, j}[n]}, \quad$ and $\quad B_{k, n}=\min _{m \in \mathcal{W}} \zeta_{m, k}^{\star}[n] \quad \forall k, n$

We note that considering constraints (33b) and (33c) jointly ensures that $\alpha_{k}[n]=0$ or $\alpha_{k}[n]=1$ must hold, similar to the original binary constraint (6), i.e., $\alpha_{k}[n] \in\{0,1\}, \forall k, n$. Following the above transformation, though the NP-hard mixedinteger user scheduling optimization problem is equivalently converted into a continuous optimization problem, it is still challenging to solve due to nonconvex linear-minus-quadratic constraint (33c). Here, we apply the first order restrictive approximation [49] to obtain a global upper-bound at the given local point $\alpha_{k}^{l o}[n], \forall n, k$ and rewrite it as

$$
\sum_{n=1}^{N} \sum_{k=1}^{K}\left[\left(1-2 \alpha_{k}^{l o}[n]\right) \alpha_{k}[n]+\left(\alpha_{k}^{l o}[n]\right)^{2}\right] \leq 0
$$

However that replacing (33c) by the convex constraint obtained in (34) converts problem (P1) into a convex optimization problem which can be sequentially solved via SCA method, due to co-existence of constraints (33b) and (33c), it is, in general, difficult to attain a feasible solution. Therefore, to tackle this issue, we apply the penalty-SCA (PSCA) technique wherein we bring the constraint (34) into the objective function via adding a penalty term. Although this violates the binary constraint but makes the problem feasible, enabling us to iteratively employ the SCA technique to solve the resulting optimization problem. To proceed, we rewrite the problem (P1) approximately as

$$
\begin{aligned}
& \text { (P1.1) : } \underset{\boldsymbol{\alpha}, \eta}{\operatorname{maximize}} \quad \psi-\mu \eta \\
& \text { s.t. } \quad(33 \mathrm{a}),(33 \mathrm{~b}),(33 \mathrm{~d}) \\
& \frac{1}{N} \sum_{n=1}^{N} A_{k, n} \alpha_{k}[n] \geq \psi, \forall k \in \mathcal{K} \\
& \sum_{n=1}^{N} \sum_{k=1}^{K}\left[\left(1-2 \alpha_{k}^{l o}[n]\right) \alpha_{k}[n]+\left(\alpha_{k}^{l o}[n]\right)^{2}\right] \leq \eta,
\end{aligned}
$$

where $\eta$ is a non-negative slack variable, and $\mu$ is the given penalty parameter. It is worth stressing that feasible set of problem $(\mathrm{P} 1.1)$ is larger than that of $(\mathrm{P} 1)$; therefore, by choosing a small initial value for $\mu$ we can make problem (P1.1) feasible, and then by gradually increasing $\mu$, or in some sense, forcing $\eta$ to approach zero via maximizing the given objective function, we can reach the optimal solution of (P1.1) which can serve as the optimal solution for (P1) once $\eta=0$ is attained. Note that problem (P1.1) is indeed a linear programming (LP) that can be readily handled in polynomial time order via a convex optimization toolbox, e.g., CVX [50].

\section{B Sub-problem II: Joint Power Allocation Optimization}

In this subsection, we tackle the optimization of network power allocations. To this end, the corresponding sub-problem for jointly optimizing AP's transmission power $\mathbf{P}_{a}$ as well as maximum AN transmissions of UCJ $\mathbf{P}_{j}$ can be given as

$$
\begin{aligned}
& \text { (P2) : } \underset{\mathbf{P}_{a}, \hat{\mathbf{P}}_{j}}{\operatorname{maximize}} \min _{k \in \mathcal{K}} \frac{1}{N} \sum_{n=1}^{N} A_{k, n} \ln \left(1+\frac{B_{k, n} p_{a}[n]}{C_{k, n} \hat{p}_{j}[n]+1}\right) \\
& \text { s.t. (19), } \\
& \sum_{k=1}^{K} \alpha_{k}[n] \min _{m \in \mathcal{W}}\left(1-D_{n, k, m} \frac{p_{a}[n]}{p_{j}[n]}\right) \geq 1-\varepsilon, \forall n \in \mathcal{N} \quad \text { (36b) }
\end{aligned}
$$

where, for $\forall n \in \mathcal{N}, \forall k \in \mathcal{K}, \forall m \in \mathcal{W}$,

$$
\begin{gathered}
A_{k, n}=\frac{\mathrm{W} \alpha_{k}[n]}{N \ln (2)\left(P_{f, r}[n]+P_{f, j}[n]\right)}, B_{k, n}=\frac{g_{a r k}[n]}{\sigma_{k}^{2}[n]}, \\
C_{k, n}=\frac{g_{j k}[n]}{2 \sigma_{k}^{2}[n]}, D_{n, k, m}=\frac{g_{a r m}[n]}{g_{j m}[n]}
\end{gathered}
$$

Sub-problem (P2) is nonconvex due to nonconvex objective function and constraint (36b). First, we tackle the nonconvex 
constraint (36b) by introducing non-negative slack variables $\mathbf{S}=\left\{s_{k}[n], \forall k \in \mathcal{K}, \forall n \in \mathcal{N}\right\}$, we rewrite (P2) as

$$
\begin{gathered}
\text { (P2.1) : } \underset{\mathbf{P}_{a}, \hat{\mathbf{P}}_{j}, \mathbf{S}}{\operatorname{maximize}} \min _{k \in \mathcal{K}} \sum_{n=1}^{N} A_{k, n} \ln \left(1+\frac{B_{k, n} p_{a}[n]}{C_{k, n} \hat{p}_{j}[n]+1}\right) \\
\text { s.t. } \quad(36 \mathrm{a}), \sum_{k=1}^{K} \alpha_{k}[n] s_{k}[n] \geq 1-\varepsilon, \forall n \in \mathcal{N} \\
\ln \left(\hat{p}_{j}[n]\right)+\ln \left(1-s_{k}[n]\right) \geq \ln \left(D_{n, k, m} p_{a}[n]\right), \\
\forall n \in \mathcal{N}, k \in \mathcal{K}, m \in \mathcal{W}
\end{gathered}
$$

The obtained formulation (P2.1) is in good shape but still nonconvex due to the nonconvex objective function and (38). Since the summation terms of the objective function of problem (P2.1) are in the form of concave-minus-concave owing to the fact that the logarithm of any non-negative affine function is concave, we substitute the objective function with a nonnegative slack variable $\eta$, then replacing the sum of logarithmic functions with their corresponding global concave lower bound, as well as replacing (38) with the approximate convex constraint using the first-order restrictive law of Taylor approximation at the given local point $\hat{\mathbf{P}}_{j}^{l o}=\left\{\hat{p}_{j}^{l o}[n], \forall n \in \mathcal{N}\right\}$, we can rewrite (P2.1) as the following convex reformulation

$$
\begin{array}{ll}
(\mathrm{P} 2.2): \underset{\mathbf{P}_{a}, \hat{\mathbf{P}}_{j}, \mathbf{S}, \eta}{\operatorname{maximize}} & \eta \\
\text { s.t. } \quad & \frac{1}{N} \sum_{n=1}^{N} A_{k, n}\left[\ln \left(1+B_{k, n} p_{a}[n]+C_{k, n} \hat{p}_{j}[n]\right)\right. \\
& \left.-f_{2}\left(\hat{p}_{j}[n], \hat{p}_{j}^{l o}[n]\right)\right] \geq \eta, \forall k \in \mathcal{K} \\
\text { (37), } & \ln \left(\hat{p}_{j}[n]\right)+\ln \left(1-s_{k}[n]\right) \geq g_{2}\left(p_{a}[n], p_{a}^{l o}[n]\right), \\
& \forall n \in \mathcal{N}, k \in \mathcal{K}, m \in \mathcal{W}
\end{array}
$$

where

$$
\begin{array}{r}
f_{2}\left(\hat{p}_{j}[n], \hat{p}_{j}^{l o}[n]\right)=\ln \left(1+C_{k, n} \hat{p}_{j}^{l o}[n]\right) \\
+\frac{C_{k, n}\left(\hat{p}_{j}[n]-\hat{p}_{j}^{l o}[n]\right)}{1+C_{k, n} \hat{p}_{j}^{l o}[n]}, \forall n \in \mathcal{N}, k \in \mathcal{K} \\
g_{2}\left(p_{a}[n], p_{a}^{l o}[n]\right)=\ln \left(D_{n, k, m} p_{a}^{l o}[n]\right) \\
+\frac{p_{a}[n]-p_{a}^{l o}[n]}{p_{a}^{l o}[n]}, \forall n \in \mathcal{N}, k \in \mathcal{K}, m \in \mathcal{W}
\end{array}
$$

Since problem (P2.2) is a conic convex optimization problem, it can be efficiently solved using CVX.

Note that, with more relaxations, a joint design of user scheduling and network power allocation can be presented at the cost of relatively higher complexity than individual design as given in Appendix A.

\section{Sub-problem III: Beamforming optimization}

To optimize beamforming matrices, we can write the corresponding subproblem to optimize $\boldsymbol{\Phi}$, given the other variables fixed, as

$$
\begin{gathered}
\text { (P3): } \underset{\boldsymbol{\Phi}}{\operatorname{maximize}} \min _{k \in \mathcal{K}} \frac{\sum_{n} A_{k, n} \ln \left(1+B_{k, n}\left\|\mathbf{e}_{r k}^{\dagger}[n] \Phi[n] \mathbf{e}_{a r}[n]\right\|^{2}\right)}{N} \\
\text { s.t. } \quad \sum_{k=1}^{K} \alpha_{k}[n] \min _{m \in \mathcal{W}}\left[1-C_{m, k, n}\left\|\mathbf{e}_{r m}^{\dagger}[n] \Phi[n] \mathbf{e}_{a r}[n]\right\|^{2}\right] \\
\geq 1-\varepsilon, \forall n \in \mathcal{N} \\
0 \leq \rho_{n}[l] \leq 1, \forall l \in \mathcal{L}, n \in \mathcal{N} \\
0 \leq \phi_{n}[l] \leq 2 \pi, \forall l \in \mathcal{L}, n \in \mathcal{N}
\end{gathered}
$$

where $\boldsymbol{\rho}=\left\{\rho_{n}[l], \forall l \in \mathcal{L}, n \in \mathcal{N}\right\}$ and $\boldsymbol{\phi}=\left\{\phi_{n}[l], \forall l \in\right.$ $\mathcal{L}, n \in \mathcal{N}\}$, and for $\forall n \in \mathcal{N}, k \in \mathcal{K}, m \in \mathcal{W}$

$$
\begin{gathered}
A_{k, n}=\frac{\alpha_{k}[n] \mathrm{W}}{P_{f, r}[n]+P_{f, j}[n]}, \quad B_{k, n}=\frac{p_{a}[n]\left\|\tilde{h}_{a r k}[n]\right\|^{2}}{\frac{1}{2} \hat{p}_{j}[n] g_{j k}+\sigma_{k}^{2}[n]}, \\
C_{m, k, n}=\frac{p_{a}[n]\left\|\tilde{h}_{a r m}[n]\right\|^{2}}{\hat{p}_{j}[n] g_{j m}},
\end{gathered}
$$

Now, exploiting the change of variable technique and defining the slack vector $\mathbf{u}[n]=\left[u_{1}[n], u_{2}[n], \cdots, u_{L}[n]\right]^{\mathrm{T}}$ with $u_{l}[n]=\rho_{l}[n] \exp \left(-j \phi_{l}[n]\right), \forall l \in \mathcal{L}, n \in \mathcal{N}$, and taking the auxiliary column vectors $\mathbf{a}_{k}[n]=\operatorname{diag}\left(\mathbf{e}_{r k}^{\dagger}[n]\right) \mathbf{e}_{a r}[n], \forall k \in$ $\mathcal{K}, n \in \mathcal{N}, \mathbf{b}_{m}[n]=\operatorname{diag}\left(\mathbf{e}_{r m}^{\dagger}[n]\right) \mathbf{e}_{a r}[n]$, we can reformulate (P3), introducing the slack variables $\boldsymbol{\eta}=\left\{\eta_{k}[n], \forall n \in\right.$ $\mathcal{N}, k \in \mathcal{K}\}$ as

$$
\begin{aligned}
& \text { (P3.1) : } \underset{\boldsymbol{\eta}, \mathbf{u}}{\operatorname{maximize}} \min _{k \in \mathcal{K}} \frac{1}{N} \sum_{n=1}^{N} A_{k, n} \ln \left(1+B_{k, n} \eta_{k}[n]\right) \\
& \text { s.t. } \quad \mathbf{u}^{\dagger}[n] \mathbf{A}_{k}[n] \mathbf{u}[n] \geq \eta_{k}[n], \quad \forall n \in \mathcal{N}, k \in \mathcal{K}
\end{aligned}
$$

$$
\begin{gathered}
\sum_{k=1}^{K} \alpha_{k}[n] \min _{m \in \mathcal{W}}\left[1-C_{m, k, n} \mathbf{u}^{\dagger}[n] \mathbf{B}_{m}[n] \mathbf{u}[n]\right] \\
\geq 1-\varepsilon, \forall n \in \mathcal{N} \\
\|\mathbf{u}[n]\| \leq 1, \quad \forall n \in \mathcal{N}
\end{gathered}
$$

where $\mathbf{A}_{k}[n]=\mathbf{a}_{k}[n] \mathbf{a}_{k}^{\dagger}[n], \mathbf{B}_{m}[n]=C_{m, k, n} \mathbf{b}_{m}[n] \mathbf{b}_{m}^{\dagger}[n]$.

Note that the objective function is in the form of the nonnegative combination of logarithms, each of which is nondecreasing w.r.t $\eta_{k}[n]$. Thus the inequality (44a) must be met with equality at the optimal point; otherwise, the objective value can be further improved, violating the optimality. Problem (P3.1) is a quadratically constrained nonlinear program (QCNLP) which is NP-hard, so is its relaxed version: quadratically constrained quadratic programming (QCQP). Therefore, to facilitate the development of problem, we define $N$ matrices of size $L$-by- $L$, i.e., $\mathbb{W} \triangleq\left\{\mathbf{W}[n] \triangleq\left\{\mathbf{u}[n] \mathbf{u}^{\dagger}[n], \forall n \in \mathcal{N}\right\}\right.$, 
which ensures $\mathbb{W}$ to be rank one Hermitian positive semidefinite (PSD) matrix set. Then we recast (P3.1) as

$$
\begin{gathered}
\text { (P3.2) : } \underset{\boldsymbol{\eta}, \mathbb{W}}{\operatorname{maximize}} \min _{k \in \mathcal{K}} \frac{1}{N} \sum_{n=1}^{N} A_{k, n} \ln \left(1+B_{k, n} \eta_{k}[n]\right) \\
\text { s.t. } \quad \operatorname{tr}\left(\mathbf{A}_{k}[n] \mathbf{W}[n]\right) \geq \eta_{k}[n], \forall n \in \mathcal{N}, k \in \mathcal{K} \\
\sum_{k=1}^{K} \alpha_{k}[n] \min _{m \in \mathbf{W}}\left[1-C_{m, k, n} \operatorname{tr}\left(\mathbf{B}_{m}[n] \mathbf{W}[n]\right)\right] \\
\geq 1-\varepsilon, \forall n \in \mathcal{N} \\
\mathbf{W}_{l, l}[n] \leq 1, \forall l \in \mathcal{L}, n \in \mathcal{N} \\
\mathbf{W}[n] \succeq 0, \forall n \in \mathcal{N} \\
\operatorname{rank}(\mathbf{W}[n])=1, \forall n \in \mathcal{N}
\end{gathered}
$$

where $\mathbf{W}_{l, l}[n]$ in (45c) refer to the elements alongside the main diagonal of the matrix of $\mathbf{W}[n]$ and is the reformulation of the magnitude constraint (44c), (45d) is the linear matrix inequality (LMI) indicating that $\mathbf{W}[n] \in \mathbb{S}^{+}$and this constraint is convex w.r.t the optimization variables. It is worth pointing out that by such reformulation, we have converted nonconvex objective constraints in (44) into convex equivalent constraints, but unfortunately (P3.2) is as hard as (P3.1) to solve due to the newly introduced nonconvex rank constraint (45e). Nonetheless, dropping such nonconvex constraint, we can obtain the semi-definite relaxation (SDR) reformulation of (45) as

$$
\begin{gathered}
(\mathrm{P} 3.2 .1): \underset{\boldsymbol{\eta}, \mathbb{W}}{\operatorname{maximize}} \min _{k \in \mathcal{K}} \frac{1}{N} \sum_{n=1}^{N} A_{k, n} \ln \left(1+B_{k, n} \eta_{k}[n]\right) \\
\text { s.t. } \quad(45 \mathrm{a}),(45 \mathrm{~b}),(45 \mathrm{c}),(45 \mathrm{~d})
\end{gathered}
$$

Note that problem (P3.2.1) is a convex semi-definite programming (SDP), which can be solved in a numerically reliable and efficient manner by applying the interior-point method [51]. Though SDR is a computationally efficient approximation approach, problem (P3.2.1) does not necessarily generate rankone matrices as its optimal solution $\mathbb{W}^{\star}=\left\{\mathbf{W}^{\star}[n], \forall n \in \mathcal{N}\right\}$. Indeed, if $\mathbf{W}^{\star}[n]$ for $\forall n \in \mathcal{N}$ are of rank one, then we can readily write $\mathbf{W}^{\star}[n]=\mathbf{u}^{\star}[n] \mathbf{u}^{\star \dagger}[n]\left(\mathbf{u}^{\star}[n], \forall n \in \mathcal{N}\right.$ being the eigenvector corresponding the only non-zero eigenvalue of $\mathbf{W}^{\star}[n]$ ) extracting not only feasible but also optimal solution $\mathbf{u}^{\star}[n]$ to problem (P3.1) which is consequently the optimal solution to problem (P3). Otherwise, if the rank of $\mathbf{W}^{\star}[n]$ is larger than one, the SDR method can only generate a tight lower-bound on the optimal objective value of (P3.2). To this end, we proceed to tackle this issue by employing an iterative rank minimization approach, namely rank-penalty SCA (RPSCA), as discussed below.

The essential notion of RP-SCA technique lies in the fact that every rank-one matrix has only one non-zero eigenvalue. Hence, instead of making constraints on the rank, we focus on the $L-1$ smallest eigenvalues of $\mathbf{W}[n], \forall n$ forcing them to be all zero via an efficient iterative approach, as discussed in the lemma below.
Lemma 1. Let $\mathbf{W} \in \mathbb{S}^{+}$be a nonzero PSD matrix, then

$$
\operatorname{rank}(\mathbf{W})=1 \Longleftrightarrow \psi I-\mathbf{V}^{\dagger} \mathbf{W} \mathbf{V} \succeq 0,
$$

where $\psi=0, \mathbf{I}$ is the identity matrix of size $(L-1)$, and $\mathbf{V} \in$ $\mathbb{C}^{L \times L-1}$ are the eigenvectors corresponding to the $(L-1)$ smallest eigenvalues of $\mathbf{W}$.

Proof. Since W is PSD, it is Hermitian with nonnegative eigenvalues (please see Appendix B). We assume that the nonnegative eigenvalues of $\mathbf{W}$ are sorted in ascending order as $\left[\lambda_{1}, \lambda_{2}, \cdots, \lambda_{L}\right]$ with the $(L-1)$ corresponding normalized eigenvectors $\mathbf{V}=\left[\mathbf{v}_{1}, \mathbf{v}_{2}, \cdots, \mathbf{v}_{L-1}\right]$. Since the Rayleigh quotient of an eigenvector equals with its associated eigenvalue, i.e., $\mathbf{v}_{l}^{\dagger} \mathbf{W} \mathbf{v}_{l}=\lambda_{l}, \forall l$ and eigenvectors of distinct eigenvalues for any Hermitian matrix are orthogonal, thus $\psi I-\mathbf{V}^{\dagger} \mathbf{W} \mathbf{V}=\operatorname{diag}\left(\psi-\lambda_{1}, \psi-\lambda_{2}, \cdots, \psi-\lambda_{L-1}\right)$ is a diagonal matrix. Therefore, $\mathbf{W}$ is rank one iff all the diagonal elements of $\psi I-\mathbf{V}^{\dagger} \mathbf{W} \mathbf{V}$ are all zero given $\psi=0$.

Using Lemma 1, we replace the rank constraint (45e) in (P2.2) with the corresponding semi-definite constraint and introduce the slack variables $\nu$ and $\boldsymbol{\psi}=\{\psi[n], n \in \mathcal{N}\}$, then rewrite (P3.2) as

$$
\begin{aligned}
(\mathrm{P} 3.3) & \underset{\nu, \boldsymbol{\psi}, \boldsymbol{\eta}, \mathbb{W}}{\operatorname{maximize}} \nu-\sum_{n=1}^{N} \mu \psi[n] \\
\text { s.t. } & \frac{1}{N} \sum_{n=1}^{N} A_{k, n} \ln \left(1+B_{k, n} \eta_{k}[n]\right) \geq \nu, \forall k \in \mathcal{K} \\
& \psi[n] I-\mathbf{V}[n]^{\dagger} \mathbf{W}[n] \mathbf{V}[n] \succeq 0, \forall n \in \mathcal{N} \\
& \boldsymbol{\psi} \geq \mathbf{0},(46 \mathrm{a})
\end{aligned}
$$

It is worth mentioning that directly applying rank-one reformulation in Lemma 1 likely results in an infeasible problem. Therefore, by introducing a slack variable and bringing the rank-penalty to the objective function with weighting factor $\mu$, we attempt to iteratively maximize the original objective value while minimizing the rank penalty term. Since $\mathbf{W}[n] \in$ $\mathbb{S}^{+}, \forall n$ then $\mathbf{V}^{\dagger} \mathbf{W} \mathbf{V} \in \mathbb{S}^{+}\left(\left(\mathbf{V}^{\dagger} \mathbf{W} \mathbf{V}\right)^{\dagger}=\mathbf{V}^{\dagger} \mathbf{W V}\right)$. Thus, $\psi$ needs to be sufficiently large and nonnegative to make the problem feasible. Therefore, less focus initially is on the rankone requirement, but rank one is satisfied when $\psi=0$. Once the rank-one PSD matrix $\mathbf{W}^{\star}$ is obtained, the optimal solution of (P3.1) $\mathbf{u}^{\star}$ can be computed via $\mathbf{u}^{\star}=\sqrt{\lambda_{L}} \mathbf{v}_{L}$, where $\lambda_{L} \in \mathbb{R}^{+}$is the largest eigenvalue of $\mathbf{W}^{\star}$ and $\mathbf{v}_{L} \in \mathbb{C}^{L}$ is the corresponding normalized eigenvector. We summarize the proposed beamforming maximization in Algorithm 1.

Now we present a low-complex alternative design to balance mAEE performance and computational complexity. Note that with the known scheduled UE per time slot, the fixed transmit powers and UAVs' trajectories, we can maximize the quality of the received signal at Bob via coherently combing signals from 


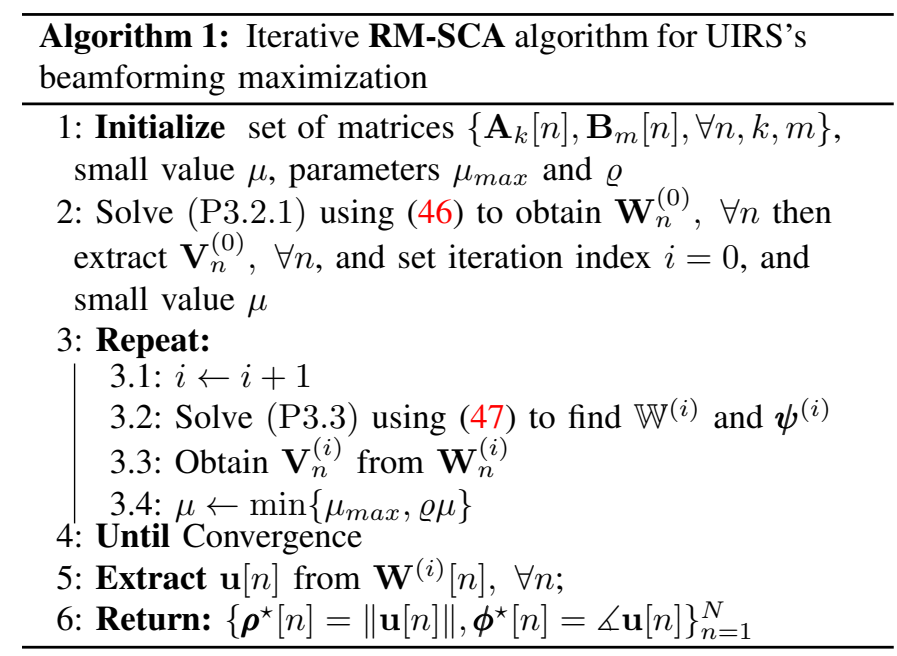

different paths, meanwhile degrading the leakage to Willies. Given UE $k$ is scheduled per time slot, we can rewrite (14) as

$$
\begin{aligned}
& h_{\text {ark }}[n]=\tilde{h}_{\text {ark }}[n] \\
& \times \sum_{l=1}^{L} \rho_{l}[n] \mathrm{e}^{j\left(\phi_{l}[n]+\beta_{l}^{k}[n]-\theta_{l}^{k}[n]\right)}, \forall k \in \mathcal{K}, n \in \mathcal{N}
\end{aligned}
$$

Note that improving received signal energy at Bob via improving the channel gain (48) leads to mAEE improvement according to (20) and (32). Thus, by properly aligning the phases of different paths, we can improve the channel quality by setting $\phi_{l}[n]+\beta_{l}^{k}[n]-\theta_{l}^{k}[n]=\omega, \rho_{l}^{\star}[n]=1 . \forall l \in \mathcal{L}, n \in \mathcal{N}$, where $0 \leq \omega \leq 2 \pi$, the optimized IRS beamforming matrices can be obtained as

$$
\Phi^{\star}[n]=\operatorname{diag}\left(\left\{\rho_{l}^{\star}[n] \mathrm{e}^{j \phi_{l}^{\star}[n]}\right\}_{l=1}^{L}\right), \forall l \in \mathcal{L}, n \in \mathcal{N}
$$

with

$$
\phi_{l}^{\star}[n]=\omega+\theta_{l}^{k}[n]-\beta_{l}^{k}[n], \quad \rho_{l}^{\star}[n]=1,
$$

Here, the magnitude of the IRS reflected beam needs to be set unity, i.e., $\rho_{l}[n]=1, \forall l \in \mathcal{L}, n \in \mathcal{N}$; otherwise $\rho_{l}[n]$ can be increased, resulting in further improvement of $h_{\text {ark }}[n]$ and then our objective function in terms of the mAEE. This demonstrates the sub-optimality of the low-complex design. Consequently, (48) is maximized and accordingly $\bar{R}_{k}^{l b}[n]$ given by (20) can be simplified as

$\bar{R}_{k}^{l b}[n]=\mathrm{W} \log _{2}\left[1+\frac{p_{a}[n]\left(\frac{L^{2} h_{r 0}[n] \mathrm{e}^{-\kappa\left(\left\|\mathbf{q}_{r}[n]-\mathbf{q}_{a}\right\|+\left\|\mathbf{q}_{r}[n]-\mathbf{q}_{k}\right\|\right)}}{\left\|\mathbf{q}_{r}[n]-\mathbf{q}_{a}\right\| \rho\left\|\mathbf{q}_{r}[n]-\mathbf{q}_{k}\right\| \rho}\right)}{\frac{1}{2} \hat{p}_{j}[n] \frac{h_{j 0}[n] \mathrm{e}^{-\kappa\left\|\mathbf{q}_{j}[n]-\mathbf{q}_{k}\right\|}}{\left\|\mathbf{q}_{j}[n]-\mathbf{q}_{k}\right\| \rho}+1}\right]$

where $h_{r 0}[n] \triangleq \frac{\lambda_{c}^{2}}{64 \pi^{3} \sigma_{k}^{2}[n]}$ and $h_{j 0}[n] \triangleq \frac{\lambda_{c}^{2}}{16 \pi^{2} \sigma_{k}^{2}[n]}$. Furthermore, following the above beamforming design, the minimum detection error rate given by (30) can be rewritten as

$$
\begin{gathered}
\zeta_{m, k}^{\star}[n]=1-\frac{p_{a}[n] \frac{\tilde{L}[n] h_{r 0} \exp \left(-\kappa\left(\left\|\mathbf{q}_{r}[n]-\mathbf{q}_{a}\right\|+\left\|\mathbf{q}_{r}[n]-\mathbf{q}_{m}\right\|\right)\right)}{\left\|\mathbf{q}_{r}[n]-\mathbf{q}_{a}\right\|^{\rho}\left\|\mathbf{q}_{r}[n]-\mathbf{q}_{m}\right\|^{\rho}}}{\hat{p}_{j}[n] \frac{h_{j 0} \exp \left(-\kappa\left\|\mathbf{q}_{j}[n]-\mathbf{q}_{m}\right\|\right)}{\left\|\mathbf{q}_{j}[n]-\mathbf{q}_{m}\right\|^{\rho}}} \\
\forall n \in \mathcal{N}, k \in \mathcal{K}, m \in \mathcal{W}
\end{gathered}
$$

where

$$
\tilde{L}[n] \triangleq\left\|\sum_{n=1}^{L} \exp \left(j\left(\phi_{l}^{\star}[n]-\theta_{l}^{m}[n]+\beta_{l}^{m}[n]\right)\right)\right\|^{2}
$$

Since (51) is too complicated, we consider using its restrictive conservative lower-bound, leading to the reformulation of (31) as

$$
\begin{aligned}
\widetilde{\mathrm{C}} 7: \sum_{k=1}^{K} \alpha_{k}[n] & \min _{m \in \mathcal{W}}\left[1-\frac{p_{a}[n]}{\hat{p}_{j}[n]}\right. \\
& \left.\times \frac{\frac{L^{2} h_{r 0} \exp \left(-\kappa\left(\left\|\mathbf{q}_{r}[n]-\mathbf{q}_{a}\right\|+\left\|\mathbf{q}_{r}[n]-\mathbf{q}_{m}\right\|\right)\right)}{\left\|\mathbf{q}_{r}[n]-\mathbf{q}_{a}\right\| \rho\left\|\mathbf{q}_{r}[n]-\mathbf{q}_{m}\right\| \rho}}{\frac{\left.h_{j 0} \exp (-\kappa) \mathbf{q}_{j}[n]-\mathbf{q}_{m} \|\right)}{\left\|\mathbf{q}_{j}[n]-\mathbf{q}_{m}\right\| \rho}}\right] \\
& \geq 1-\varepsilon, \forall n \in \mathcal{N}, k \in \mathcal{K}
\end{aligned}
$$

In the sequel, we utilise above reformulations for trajectory and velocity optimization.

\section{Sub-problem IV: Joint UCJ's Trajectory and Velocity Optimization}

We focus on jointly optimizing the UCJ's trajectory $\mathbf{q}_{j}$ and velocity $\mathbf{v}_{j}$ while keeping the transmit powers, UIRS's beamforming matrix, and user scheduling $\boldsymbol{\alpha}$ fixed. The corresponding sub-problem is given in (53a) (shown on top of the next page), where

$$
\begin{gathered}
A_{k, n}=\frac{\alpha_{k}[n] W}{\ln (2)}, B_{k, n}=\frac{p_{a}[n] g_{a r k}[n]}{\sigma_{k}^{2}}, C_{k, n}=\left(\frac{\lambda_{c}}{4 \pi}\right)^{2} \frac{\hat{p}_{j}[n]}{2 \sigma_{k}^{2}} \\
D_{m, k, n}=\frac{p_{a}[n] g_{a r m}[n]}{p_{j}[n]\left(\frac{\lambda_{c}}{4 \pi}\right)^{2}}, \forall n \in \mathcal{N}, k \in \mathcal{K}, m \in \mathcal{W}
\end{gathered}
$$

Problem (P4) is nonconvex being in the form of minimum of sum-of-fractional-nonlinear-programming. To facilitate the development of optimization framework, we first mention the following lemma.

Lemma 2. Given $N$ pairs of non-negative functions $f_{n}(\mathbf{x})$ and positive functions $g_{n}(\mathbf{x})$, as well as having a nonempty constraint set $\mathcal{X}$, the general average-of-ratios fractional programming (FP) maximization can be represented in the form of

$$
\begin{aligned}
& \underset{\mathbf{x}}{\operatorname{maximize}} \frac{1}{N} \sum_{n=1}^{N} \frac{f_{n}(\mathbf{x})}{g_{n}(\mathbf{x})} \\
& \text { s.t. } \quad \mathbf{x} \in \mathcal{X}
\end{aligned}
$$

The above problem is in general nonconvex, thereby too difficult to solve. However, if we could obtain a tight concave lower-bounds on $f_{n}(\mathbf{x}), \forall n$, represented by $f_{n}^{l b}(\mathbf{y}), \forall n$, and the tight convex upper-bounds of $g_{n}(\mathbf{x}), \forall n$, denoted as $g_{n}^{u p}(\mathbf{x}), \forall n$, such that $f_{n}(\mathbf{x}) \geq f_{n}^{l b}(\mathbf{y})$ and $g_{n}(\mathbf{x}) \leq$ $g_{n}^{u p}(\mathbf{y}), \forall n$, then by applying the Dinkelbach-based quadratic transformation introduced in [52], (54) can be approximately reformulated as a convex optimization problem given by

$$
\begin{aligned}
& \underset{\mathbf{y}, \boldsymbol{\gamma}}{\operatorname{maximize}} \frac{1}{N} \sum_{n=1}^{N}\left(2 \gamma_{n}^{2} \sqrt{f_{n}^{l b}(\mathbf{y})}-\gamma_{n}^{2} g_{n}^{u p}(\mathbf{y})\right) \\
& \text { s.t. } \mathbf{y} \in \mathcal{Y} \subseteq \mathcal{X}
\end{aligned}
$$




$$
\begin{gathered}
\text { (P4) : } \underset{\mathbf{q}_{j}, \mathbf{v}_{j}}{\operatorname{maximize}} \min _{k \in \mathcal{K}} \frac{1}{N} \sum_{n=1}^{N} \frac{A_{k, n} \ln \left(1+\frac{B_{k, n}}{C_{k, n} \frac{\exp \left(-\kappa\left\|q_{j}[n]-\mathbf{q}_{k}\right\|\right)}{\left\|\mathbf{q}_{j}[n]-\mathbf{q}_{k}\right\| \rho}}\right)}{P_{o}\left(1+c_{0}\left\|\mathbf{v}_{j}[n]\right\|^{2}\right)+c_{1}\left\|\mathbf{v}_{j}[n]\right\|^{3}+P_{i}\left(\sqrt{1+c_{2}^{2}\left\|\mathbf{v}_{j}[n]\right\|^{4}}-c_{2} \mathbf{v}_{j}[n] \|^{2}\right)^{\frac{1}{2}}+P_{f, r}[n]} \\
\text { s.t. } \quad(4), \sum_{k=1}^{K} \alpha_{k}[n] \min _{m \in \mathcal{W}}\left\{1-D_{m, k, n}\left\|\mathbf{q}_{j}[n]-\mathbf{q}_{m}\right\|^{\rho} \exp \left(\kappa\left\|\mathbf{q}_{j}[n]-\mathbf{q}_{m}\right\|\right)\right\} \geq 1-\varepsilon, \forall n \in \mathcal{N}, m \in \mathcal{W}
\end{gathered}
$$

where $\gamma=\left\{\gamma_{n}\right\}_{n=1}^{N}$ are auxiliary variables whose optimal values with fixed $\mathbf{y}$ can be obtained as $\gamma^{\star}=\frac{\sqrt{f_{n}^{2 b}(\mathbf{y})}}{g_{n}^{u n}(\mathbf{y})}$. It is also worth stressing that the optimal value of problem (54) is no less than that of (55). Since problem (55) is a convex optimization problem; thus, it can be solved efficiently by alternatively updating $\gamma$ and $\mathbf{y}$ starting from a feasible point. Consequently, having solved (55), we can achieve an approximate solution of the original problem given by (54) but with guaranteed convergence.

Using Lemma 2, we first convert each ratio of summation terms in the objective function of $(\mathrm{P} 4)$ to be in the form of non-negative concave over positive convex functions. To this end, we take non-negative slack variables $\mathbf{w}=\left\{w_{k}[n], \forall n \in\right.$ $\mathcal{N}, k \in \mathcal{K}\}, \mathbf{t}=\{t[n], \forall n \in \mathcal{N}\}$ such that

$$
w_{k}[n]=\frac{\exp \left(-\kappa\left\|q_{j}[n]-\mathbf{q}_{k}[n]\right\|\right)}{\left\|\mathbf{q}_{j}[n]-\mathbf{q}_{k}[n]\right\|^{\rho}}, \forall n \in \mathcal{N}, k \in \mathcal{K}
$$

and

$$
t[n]=\left(\sqrt{1+c_{2}^{2}\left\|\mathbf{v}_{j}[n]\right\|^{4}}-c_{2}\left\|\mathbf{v}_{j}[n]\right\|^{2}\right)^{\frac{1}{2}}, \forall n \in \mathcal{N}
$$

Since

$$
f_{4}(w[n]) \triangleq A_{k, n} \ln \left(1+\frac{B_{k, n}}{C_{k, n} w_{k}[n]+1}\right)
$$

is convex w.r.t $w_{k}[n]$, according to [11, Lemma 1], we can apply the first order restrictive approximation to obtain a global concave lower-bound at the given local point $\mathbf{w}^{l o}=$ $\left\{w_{k}^{l o}[n], \forall n \in \mathcal{N}, k \in \mathcal{K}\right\}$ as

$$
\begin{aligned}
& f_{4}\left(w_{k}[n]\right) \geq f_{4}\left(w_{k}^{l o}[n]\right) \\
& -\frac{A_{k, n} B_{k, n} C_{k, n}\left(w_{k}[n]-w_{k}^{l o}[n]\right)}{\left(C_{k, n} w_{k}^{l o}[n]+1\right)\left(C_{k, n} w_{k}^{l o}[n]+B_{k, n}+1\right)} \triangleq f_{4}^{l b}\left(w_{k}[n]\right),
\end{aligned}
$$

We reformulate problem $(\mathrm{P} 4)$, taking the new non-negative slack variables $\mathbf{v}=\left\{v_{k}[n], \forall n \in \mathcal{N}, k \in \mathcal{K}\right\}$, as

$$
\text { (P4.1) : } \underset{\mathbf{q}_{j}, \mathbf{v}_{j}, \mathbf{w}, \mathbf{v}, \mathbf{t}}{\operatorname{maximize}}
$$

$$
\min _{k \in \mathcal{K}} \frac{1}{N} \sum_{n=1}^{N}\left(2 \gamma_{k, n} \sqrt{f_{4}^{l b}\left(w_{k}[n]\right)}\right.
$$

$$
\left.-\gamma_{k, n}^{2} g_{4}^{u p}\left(\mathbf{v}_{j}[n], t[n]\right)\right)
$$

$$
\text { s.t. }
$$$$
\text { (53a), } \ln \left(w_{k}[n]\right)+\rho \ln (v[n])
$$$$
+\kappa v[n] \geq 0, \forall n \in \mathcal{N}, k \in \mathcal{K}
$$$$
v_{k}[n] \leq\left\|\mathbf{q}_{j}[n]-\mathbf{q}_{k}\right\|, \forall n \in \mathcal{N}, k \in \mathcal{K}
$$$$
t^{2}[n]+c_{2}\left\|\mathbf{v}_{j}[n]\right\|^{2} \geq \frac{1}{t^{2}[n]}, \forall n \in \mathcal{N}
$$$$
\left\|\mathbf{q}_{r}[n]-\mathbf{q}_{j}[n]\right\| \geq D_{s}, \forall n \in \mathcal{N}
$$

where $g_{4}^{u p}\left(\mathbf{v}_{j}[n], t[n]\right)$ is a convex function given by

$$
\begin{aligned}
g_{4}^{u p}\left(\mathbf{v}_{j}[n], t[n]\right) & =\left(P_{o}\left(1+c_{0}\left\|\mathbf{v}_{j}[n]\right\|^{2}\right)+c_{1}\left\|\mathbf{v}_{j}[n]\right\|^{3}\right. \\
& \left.+P_{i} t[n]+P_{f, r}[n]\right), \forall n \in \mathcal{N}
\end{aligned}
$$

Note that inequality constraints (57a), (57b), and (57c) must be met with equality at the optimal point on the grounds that the value of the objective function can be otherwise increased without violating any constraints. Note that the convexity of constraint (57a) follows from the fact that $\log (x), x>0$ is a concave function of $x$ and the sum operator preserves the convexity. Further, knowing that $h_{1}(x)=x^{\rho} \exp (\kappa x), x \geq 0$ with $\rho \geq 2$ is a convex function whose extended value extension is non-decreasing w.r.t $x$, plus $h_{2}\left(\mathbf{q}_{j}[n]\right)=\left\|\mathbf{q}_{j}[n]-\mathbf{q}_{m}\right\|$ is convex as any norm of affine function is convex, one can conclude that the composition function $h_{1} \circ h_{2}(x)$ is convex [49]. With the new reformulation, although (P4.1) is now in comparably good shape, some newly introduced constraints such as (57b), (57c) and (57d) are still non-convex. But by substituting their corresponding tight approximate convex constraints via applying the restrictive Taylor approximation at local points $\left\{\mathbf{q}_{j}^{l o}[n], t^{l o}[n], \mathbf{v}_{j}^{l o}[n], \forall n\right\}$ and introducing a slack variable $\eta_{j}$, we can reach the convex reformulation of (P4.1) given by

$$
\begin{aligned}
& \text { (P4.2): } \underset{\eta_{j}, \mathbf{q}_{j}, \mathbf{v}_{j}, \mathbf{w}, \mathbf{v}, \mathbf{t}}{\operatorname{maximize}} \eta_{j} \\
& \text { s.t. (57a), } \frac{1}{N} \sum_{n=1}^{N}\left(2 \gamma_{k, n} \sqrt{f_{4}^{l b}\left(w_{k}[n]\right)}\right. \\
& \left.-\gamma_{k, n}^{2} g_{4}^{u p}\left(\mathbf{v}_{j}[n], t[n]\right)\right) \geq \eta_{j}, \forall k \in \mathcal{K} \\
& v_{k}^{2}[n] \leq-\left\|\mathbf{q}_{j}[n]-\mathbf{q}_{k}\right\|^{2} \\
& +2\left(\mathbf{q}_{j}[n]-\mathbf{q}_{k}\right)^{\mathrm{T}}\left(\mathbf{q}_{j}[n]-\mathbf{q}_{k}\right), \quad \forall n \in \mathcal{N}, k \in \mathcal{K} \\
& -\left(t^{l o}[n]\right)^{2}+2 t[n] t^{l o}[n]+c_{2}\left(-\left\|\mathbf{v}_{j}^{l o}[n]\right\|^{2}\right. \\
& \left.+2\left(\mathbf{v}_{j}^{l o}[n]\right)^{\mathrm{T}} \mathbf{v}_{j}[n]\right) \geq \frac{1}{t^{2}[n]} ; \forall n \in \mathcal{N} \\
& 2\left(\mathbf{q}_{j}[n]-\mathbf{q}_{r}[n]\right)^{\mathrm{T}}\left(\mathbf{q}_{j}[n]-\mathbf{q}_{r}[n]\right) \\
& -\left\|\mathbf{q}_{j}[n]-\mathbf{q}_{r}[n]\right\|^{2}, \geq D_{s}^{2}, \forall n \in \mathcal{N}
\end{aligned}
$$

Note that sub-problem (P4.2), for a given $\left\{\gamma_{k, n}, k \in \mathcal{K}, n \in\right.$ $\mathcal{N}\}$, is equivalent convex approximate representation of sumof-ratios sub-problem P4 in FP form. Thus, by applying SCA we can solve problem (P4.2) as summarized in Algorithm 2. 


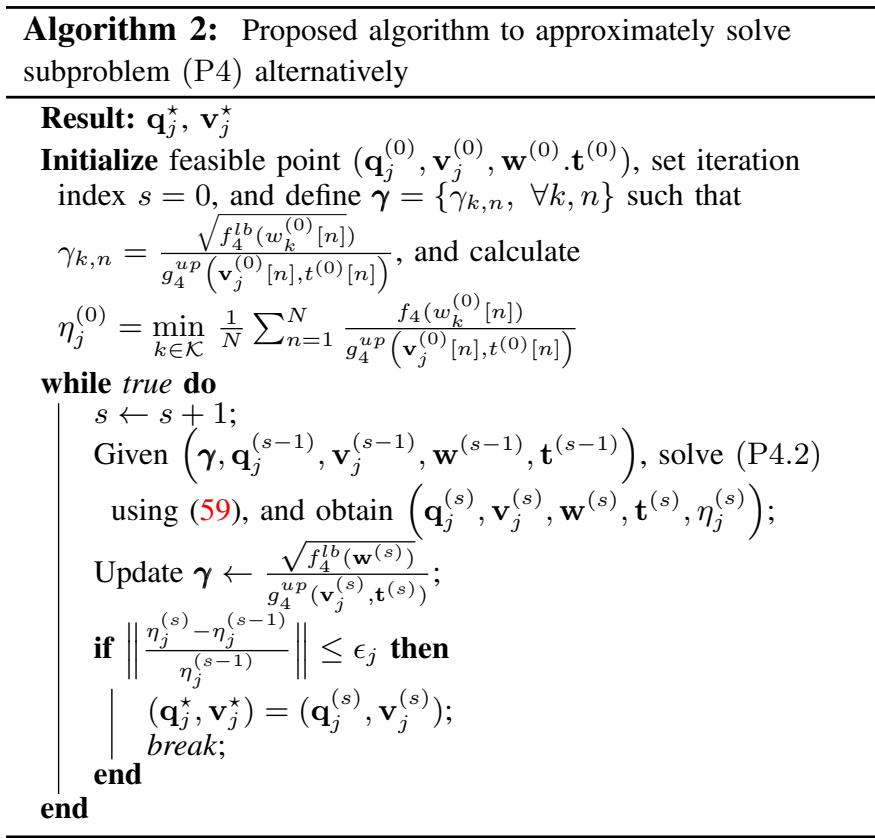

E Sub-problem V: Joint UIRS's Trajectory and Velocity Optimization

We now devise the UIRS's both trajectory $\mathbf{q}_{r}$ and velocity $\mathbf{v}_{r}$ while keeping the other variables fixed. Thus, the corresponding mAEE optimization sub-problem (P5) is introduced with constraints in (60a) where

$$
\begin{gathered}
A_{k, n}=\frac{\alpha_{k}[n] W}{\ln 2}, B_{k, n}=\frac{p_{a}[n] L^{2}}{0.5 \hat{p}_{j}[n] G_{j k}[n]+\sigma_{k}^{2}}\left(\frac{\lambda_{c}}{8 \pi \sqrt{\pi}}\right)^{2} \\
C_{m, k, n}=\frac{p_{a}[n] L^{2}}{\hat{p}_{j}[n] G_{j m}[n]}\left(\frac{\lambda_{c}}{8 \pi \sqrt{\pi}}\right)^{2}, \forall n \in \mathcal{N}, k \in \mathcal{K}, m \in \mathcal{W}
\end{gathered}
$$

Problem (P5) is nonconvex being in the form of min-of-sumof-fractional-nonlinear-programming. Similar to the approach taken to deal (P4), we can tackle (P5) by converting the FP to the quadratic subtraction form. To this end, we first mention a fruitful lemma, given below.

Lemma 3. Let $\Upsilon(x, y ; a, b, c)=\ln \left(1+a \frac{\exp (-b(x+y))}{x^{c} y^{c}}\right)$ in the domain of $x, y>0$ with constants $a, b, c \geq 0$ be a bivariate function. At a given local point $\left(x^{l o}, y^{l o}\right)$, the following inequality holds with tightness.

$$
\begin{aligned}
& \Upsilon(x, y ; a, b, c) \geq \ln \left(1+a \frac{\exp \left(-b\left(x^{l o}+y^{l o}\right)\right)}{\left(x^{l o}\right)^{c}\left(y^{l o}\right)^{c}}\right) \\
& -\frac{a\left(c+b x^{l o}\right)\left(x-x^{l o}\right)}{x^{l o}\left(a+\left(x^{l o}\right)^{c}\left(y^{l o}\right)^{c} \mathrm{e}^{b\left(x^{l o}+y^{l o}\right)}\right)} \\
& -\frac{a\left(c+b y^{l o}\right)\left(y-y^{l o}\right)}{y^{l o}\left(a+\left(x^{l o}\right)^{c}\left(y^{l o}\right)^{c} \mathrm{e}^{b\left(x^{l o}+y^{l o}\right)}\right)} \\
& \triangleq \Upsilon^{l b}\left(x, y ; x^{l o}, y^{l o}, a, b, c\right),
\end{aligned}
$$

Proof. We commence with computing the hessian of $\Upsilon(x, y)$, i.e., $\nabla^{2} \Upsilon(x, y)$. Since $\Upsilon(x, y)$ is symmetric, we shall only calculate the second order derivatives as

$$
\begin{aligned}
& \frac{\partial^{2} \Upsilon(x, y)}{\partial x^{2}}=\frac{a^{2} c+a(x y)^{c} \exp (b(x+y))\left((b x+c)^{2}+c\right)}{x^{2}\left(a+(x y)^{c} \exp (b(x+y))\right)^{2}}, \\
& \frac{\partial^{2} \Upsilon(x, y)}{\partial x \partial y}=\frac{\partial^{2} \Upsilon(x, y)}{\partial y \partial x}=\frac{a(x y)^{c}(c+b x)(c+b y)}{x y\left(a+(x y)^{c} \exp (b(x+y))\right)^{2}},
\end{aligned}
$$

Next, we derive determinant of $\nabla^{2} \Upsilon(x, y)$ as

$$
\operatorname{det}\left(\nabla^{2} \Upsilon(x, y)\right)=\frac{\partial^{2} \Upsilon(x, y)}{\partial x^{2}} \frac{\partial^{2} \Upsilon(x, y)}{\partial y^{2}}-\frac{\partial^{2} \Upsilon(x, y)}{\partial x \partial y} \frac{\partial^{2} \Upsilon(x, y)}{\partial y \partial x}
$$

$=\frac{a^{2} c\left(a c+(x y)^{c} \exp (b(x+y))\right)\left[2 c^{2}+b^{2}\left(x^{2}+y^{2}\right)+c(2 b(x+y)+1)\right]}{(x y)^{2}\left(a+(x y)^{c} \exp (b(x+y))\right)}$,

It can be readily verified that $\frac{\partial^{2} \Upsilon(x, y)}{\partial x^{2}} \geq 0$ and $\operatorname{det}\left(\nabla^{2} \Upsilon(x, y)\right) \geq 0$, implying that the hessian matrix of $\Upsilon(x, y)$ is positive semi-definite, i.e.,

$$
\nabla^{2} \Upsilon(x, y)=\left[\begin{array}{ll}
\frac{\partial^{2} \Upsilon(x, y)}{\partial x^{2}} & \frac{\partial^{2} \Upsilon(x, y)}{\partial x \partial y} \\
\frac{\partial^{2} \Upsilon(x, y)}{\partial y \partial x} & \frac{\partial^{2} \Upsilon(x, y)}{\partial y^{2}}
\end{array}\right] \succeq 0
$$

Therefore, following the second-order condition law [49], the function $\Upsilon(x, y ; a, b, c)$ is convex whose first-order Taylor approximation provides a global lower-bound at the given local point, as in (61); thus, the proof is completed.

By taking slack block variables $\mathbf{x}=\{x[n], \forall n\}$ and $\mathbf{y}=\left\{y_{k}[n] \forall k, n\right\}, \mathbf{s}=\left\{s_{k}[n], \forall k, n\right\}, \mathbf{u}=\{u[n], \forall n\}$, and leveraging Lemmas 2 and 3, (P5) can be roughly restated as

$$
\begin{aligned}
& \text { (P5.1) : } \underset{\mathbf{q}_{r}, \mathbf{v}_{r}, \mathbf{x}, \mathbf{y}, \mathbf{u}, \mathbf{s}}{\operatorname{maximize}} \eta_{r} \\
& \text { s.t. } \quad \frac{1}{N} \sum_{n=1}^{N}\left(2 \gamma_{k, n} f_{5}^{l b}-\gamma_{k, n}^{2} g_{5}^{u p}\left(\mathbf{v}_{r}[n], t[n]\right)\right) \\
& \quad \geq \eta_{r}, \forall k \in \mathcal{K} \\
& \text { (3), } \quad \sum_{k=1}^{K} \alpha_{k}[n] s_{k}[n] \geq 1-\varepsilon, \forall k \in \mathcal{K}, n \in \mathcal{N} \\
& \quad \ln \left(\frac{1-s_{k}[n]}{C_{m, k, n}}\right)+\rho\left[\ln (x[n])+\ln \left(y_{k}[n]\right)\right] \\
& +\kappa\left(x[n]+y_{k}[n]\right) \geq 0, \forall m \in \mathcal{W}, k \in \mathcal{K}, n \in \mathcal{N} \\
& \quad x[n] \geq\left\|\mathbf{q}_{r}[n]-q_{a}\right\|, \forall n \in \mathcal{N} \\
& \quad y_{k}[n] \geq\left\|\mathbf{q}_{r}[n]-q_{k}\right\|, \forall k \in \mathcal{K}, n \in \mathcal{N} \\
& u^{2}[n]+2 c_{2}\left\|v_{r}[n]\right\|^{2} \geq \frac{1}{u^{2}[n]}, \forall n \in \mathcal{N} \\
& \left\|\mathbf{q}_{r}[n]-\mathbf{q}_{j}^{l o}[n]\right\| \geq D_{s}, \forall n \in \mathcal{N}
\end{aligned}
$$

where

$$
\begin{aligned}
& f_{5}^{l b}=\sqrt{A_{k, n} \Upsilon^{l b}\left(x[n], y_{k}[n] ; x^{l o}[n], y_{k}^{l o}[n], B_{k, n}, \kappa, \rho\right)} \\
& g_{5}^{u p}\left(\mathbf{v}_{r}[n], u[n]\right)=P_{o}\left(1+c_{0}\left\|\mathbf{v}_{r}[n]\right\|^{2}\right) \\
&+c_{1}\left\|\mathbf{v}_{r}[n]\right\|^{3}+P_{i} u[n]+P_{f, j}[n],
\end{aligned}
$$




$$
\begin{gathered}
\text { (P5) : } \underset{\mathbf{q}_{r}, \mathbf{v}_{r}}{\operatorname{maximize}} \min _{k \in \mathcal{K}} \frac{1}{N} \sum_{n=1}^{N} \frac{A_{k, n} \ln \left(1+B_{k, n} \frac{\exp \left(-\kappa\left(\left\|\mathbf{q}_{r}[n]-q_{a}\right\|+\left\|\mathbf{q}_{r}[n]-\mathbf{q}_{k}\right\|\right)\right)}{\left\|\mathbf{q}_{r}[n]-q_{a}\right\| \rho\left\|\mathbf{q}_{r}[n]-q_{k}\right\|^{\rho}}\right)}{P_{o}\left(1+c_{0}\left\|\mathbf{v}_{r}[n]\right\|^{2}\right)+c_{1}\left\|\mathbf{v}_{r}[n]\right\|^{3}+P_{i}\left(\sqrt{1+c_{2}^{2}\left\|\mathbf{v}_{r}[n]\right\|^{4}}-c_{2} \mathbf{v}_{r}[n] \|^{2}\right)^{\frac{1}{2}}+P_{f, j}[n]} \\
\text { s.t. (3), } \sum_{k=1}^{K} \alpha_{k}[n] \min _{m \in \mathcal{W}}\left\{1-C_{m, n} \frac{\exp \left(-\kappa\left(\left\|\mathbf{q}_{r}[n]-q_{a}\right\|+\left\|\mathbf{q}_{r}[n]-\mathbf{q}_{k}\right\|\right)\right)}{\left\|\mathbf{q}_{r}[n]-q_{a}\right\|\left\|^{\rho}\right\| \mathbf{q}_{r}[n]-q_{k} \|^{\rho}}\right\} \geq 1-\varepsilon, \forall n \in \mathcal{N}, m \in \mathcal{W}
\end{gathered}
$$

The objective function of problem (P5.1) is now concave, since the square root of an affine function is concave and nondecreasing, therefore each term of summation is concave being in the form of concave-minus-convex, and owing to the fact that sum preserves convexity and the min function is concave and non-decreasing on every argument, thus the combination function is concave. Nonetheless, some nonconvex constraints are introduced with the above reformulation, i.e., (67f) and $(67 \mathrm{~g})$, leading problem (P5.1) to be non-convex. Hence, by substituting these nonconvex constraints by their convex approximates, we can achieve the convex reformulation of (P5.1) represented by

$$
\begin{aligned}
& \text { (P5.2) : } \underset{\eta_{r}, \mathbf{q}_{r}, \mathbf{v}_{r}, \mathbf{x}, \mathbf{y}, \mathbf{u}, \mathbf{s}}{\operatorname{maximize}} \eta_{r} \\
& \text { s.t. } \quad(67 \mathrm{a}),(67 \mathrm{~b}),(67 \mathrm{c}),(67 \mathrm{~d}),(67 \mathrm{e}) \\
& -\left(u^{l o}[n]\right)^{2}+2 u[n] u^{l o}[n]+2 c_{2}\left(-\left\|\mathbf{v}_{r}^{l o}[n]\right\|^{2}\right. \\
& \left.+2\left(\mathbf{v}_{r}^{l o}[n]\right)^{\mathrm{T}} \mathbf{v}_{r}[n]\right) \geq \frac{1}{u^{2}[n]}, \forall n \in \mathcal{N} \\
& \quad 2\left(\mathbf{q}_{r}^{l o}[n]-\mathbf{q}_{j}^{l o}[n]\right)^{\mathrm{T}}\left(\mathbf{q}_{r}[n]-\mathbf{q}_{j}^{l o}[n]\right) \\
& \quad-\left\|\mathbf{q}_{r}[n]-\mathbf{q}_{j}^{l o}[n]\right\|^{2} \geq D_{s}^{2}, \quad \forall n \in \mathcal{N}
\end{aligned}
$$

where $\left(\mathbf{q}_{j}^{l o}, \mathbf{q}_{r}^{l o}, \mathbf{v}_{r}^{l o}, \mathbf{u}^{l o}\right)$ is the given local point. Since (P5.2) is convex, it can be efficiently solved via interior-point method using CVX, providing an approximate solution to problem (P5), which is summarized in Algorithm 3.

\section{F Overall Algorithm}

Now we are ready to put together all the subsolutions and propose a low-complex sequential algorithm (see Algorithm 4) to improve mAEE of the considered scenario subject to the covertness requirement.

\section{$G$ Complexity and Convergence Analysis}

It can be mathematically proved that both the inner FP optimization and outer alternating optimization of the proposed BSCA-based algorithm are guaranteed to converge with any feasible initialization. Since the feasible solution set of $(\mathrm{P})$ is compact, its objective value is non-decreasing over iteration index, and that the optimal value of mAEE is upper bounded by a finite value. The detail is omitted for brevity, but the interested readers can refer to [13], [52]. Further, letting $\gamma_{1}, \gamma_{4}$, $\gamma_{5}$ be the maximum iteration number required for convergence of subproblems (P1.1), (P4.2), and, (P5.2), and then the worstcase computational complexity for each sub-problem and the complexity of proposed overall algorithm are obtained in Table I. It is worth noticing that the overall Algorithm 4's complexity

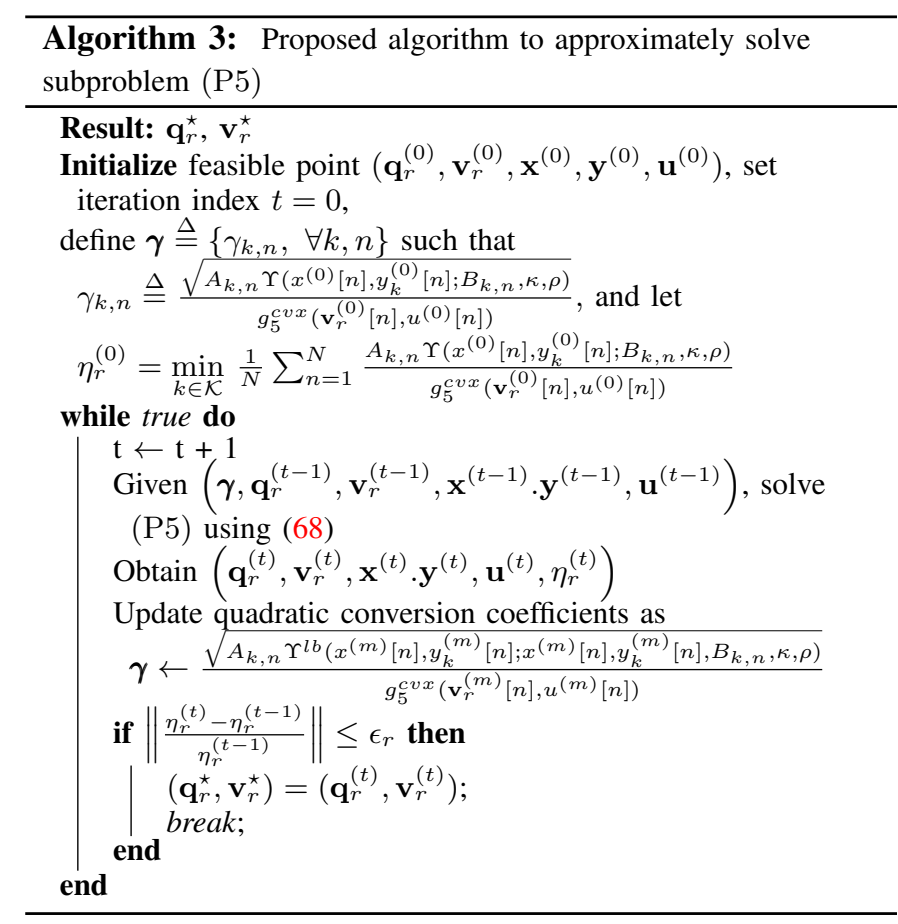

Algorithm 4: Overall proposed iterative algorithm for mAEE optimization

1: Initialize a feasible point

$\left(\boldsymbol{\alpha}^{(0)}, \mathbf{P}_{a}^{(0)}, \Phi^{(0)}, \mathbf{P}_{j}^{(0)}, \mathbf{Q}_{r}^{(0)}, \mathbf{Q}_{j}^{(0)}, \Phi^{(0)}\right)$, and set iteration index $i=0$;

2: Repeat: $i \leftarrow i+1$;

3: Solve (P1) using (35), updating $\boldsymbol{\alpha}^{(i+1)}$;

4: Given $\boldsymbol{\alpha}^{(i+1)}$, solve (P2) using (39), updating $\mathbf{P}^{(i+1)}=\left\{\mathbf{P}_{a}^{(i+1)}, \hat{\mathbf{P}}_{j}^{(i+1)}\right\}$

5: Given $\left(\boldsymbol{\alpha}^{(i+1)}, \mathbf{P}^{(i+1)}\right)$, solve (P4) via Algorithm 2, updating $\mathbf{Q}_{j}^{(i+1)}=\left\{\mathbf{q}_{j}^{(i+1)}, \mathbf{v}_{j}^{(i+1)}\right\}$

6: Given $\left(\boldsymbol{\alpha}^{(i+1)}, \mathbf{P}^{(i+1)}, \mathbf{Q}_{j}^{(i+1)}\right)$, solve (P5) via

Algorithm 3, updating $\mathbf{Q}_{r}^{(i+1)}=\left\{\mathbf{q}_{r}^{(i+1)}, \mathbf{v}_{r}^{(i+1)}\right\}$

7: Given $\left(\mathbf{Q}_{r}^{(i+1)}, \mathbf{Q}_{j}^{(i+1)}\right)$, calculate $\boldsymbol{\Phi}^{(i+1)}$ using (49)

8: Given $\left(\boldsymbol{\alpha}^{(i+1)}, \mathbf{P}^{(i+1)}, \mathbf{Q}_{j}^{(i+1)}, \mathbf{Q}_{r}^{(i+1)}, \boldsymbol{\Phi}^{(i+1)}\right)$, calculate

the value of mAEE in (32)

9: Until fractional increase of mAEE gets below the terminating threshold $\epsilon_{o}$

is in polynomial time order, demonstrating the efficiency of our proposed algorithm and suitability to the energy-constrained UAV-IoT scenarios. 
TABLE I: Complexity analysis

\begin{tabular}{lc}
\hline \hline Problem & Complexity \\
\hline User Scheduling Subproblem (P1.1) & $\mathcal{O}\left(\gamma_{1}(N K+1)(2 N K+3 N+K+2)^{\frac{3}{2}}\right)$ \\
Joint Power Allocation Subproblem (P2.2) & $\mathcal{O}\left((N(K+2)+1)^{2}(N K(K-1)+3 N+K+1)^{\frac{3}{2}}\right)$ \\
SDP relaxation IRS Subproblem (P3.2.1) & $\mathcal{O}\left(N^{4}(K+L+2)^{4}(N(K+L))^{\frac{1}{2}}\right)$ \\
RM-SCA for IRS optimization (P3.3) & $\mathcal{O}\left(\log \left(\mu_{\max } / \varrho\right)(N(K+L+4)+K)^{4}(N(K+L+1)+1)^{\frac{1}{2}}\right)$ \\
Alternative IRS beamforming Design (49) & $\mathcal{O}(L N)$ \\
UCJ's trajectory design Subproblem (P4.2) & $\mathcal{O}\left(\gamma_{4}(2 N K+5 N+1)^{2}\left(N K^{2}+2 N K+6 N+K\right)^{\frac{3}{2}}\right)$ \\
UIRS's trajectory design Subproblem (P5.2) & $\mathcal{O}\left(\gamma_{5}(2 N K+6 N+1)^{2}\left(N K^{2}+3 N K+7 N+K\right)^{\frac{3}{2}}\right)$ \\
Overall Complexity of Algorithm 4 & $\mathcal{O}\left(\left(\gamma_{4}+\gamma_{5}\right) N^{\frac{7}{2}} K^{5}+\gamma_{1}(N K)^{\frac{5}{2}}+L N\right)$ \\
\hline \hline
\end{tabular}

\section{NUMERICAL RESULTS AND DISCUSSION}

In this section, we evaluate the performance of our proposed optimization algorithm to improve the mAEE performance metric for the considered UIRS-assisted $\mathrm{THz}$ covert communication system with UCJ's cooperative jamming. To demonstrate the effectiveness of our design, we compare it with some benchmarks listed below.

- Proposed JTCD: Proposed joint trajectory and communication design for the mAEE improvement according to Algorithm 4.

- Benchmark I - CD: With fixed UAVs' trajectory and velocity block variables, only communication design is taken into account including user scheduling and transmit powers optimization $\left(\mathbf{P}_{a}^{\star}, \mathbf{P}_{j}^{\star}, \boldsymbol{\alpha}^{\star}\right)$.

- Benchmark II - TD: Keeping the communication resources fixed, joint trajectory and velocity design of both UAVs are considered $\left(\mathbf{Q}_{r}^{\star}=\left\{\mathbf{q}_{r}^{\star}, \mathbf{v}_{r}^{\star}\right\}, \mathbf{Q}_{j}^{\star}=\left\{\mathbf{q}_{j}^{\star}, \mathbf{v}_{j}^{\star}\right\}\right)$.

- Benchmark III - IFTR: Non-optimal initial feasible trajectory and resource allocations.

Unless otherwise stated, all simulation parameters are set as given follows: $v_{r}^{\max }=v_{j}^{\max }=25 \mathrm{~m} \cdot \mathrm{s}^{-1}, a_{r}^{\max }=v_{j}^{\max }=6$ $\mathrm{m} . \mathrm{s}^{-2}, f_{c}=0.3 \mathrm{THz}, \kappa=3.2094 \times 10^{-4}$ using (8), $p_{a}^{\max }=$ $p_{j}^{\max }=1 \mathrm{~W}, p_{\text {tot }}=40 \mathrm{~W}, \sigma_{k}^{2}=-180, \forall k, \mathbf{q}^{a}=[0,0,0]$, $\mathbf{q}_{r}^{I}=[100,0,50], \mathbf{q}_{j}^{I}=[80,0,50], P_{o}=79.856 \mathrm{~W}$ and $P_{i}=$ 88.63 [46], $K=5$ UEs are uniformly distributed inside a disk centered at $\mathbf{q}_{a}$ with inner and outer radii $R_{1}=100 \mathrm{~m}$ and $R_{2}=200 \mathrm{~m}$, respectively, $T=30 \mathrm{~s}, \delta_{t}=0.1 \mathrm{~s},\left(\epsilon_{o}, \epsilon_{r}, \epsilon_{j}\right)=$ $\left(10^{-1}, 10^{-3}, 10^{-3}\right) . L_{x}=5, L_{y}=6, \delta_{x}=\delta_{y}=1 \mathrm{~mm}$, $\left(c_{0}, c_{1}, c_{2}\right)=\left(2.0833 \times 10^{-4}, 0.0092,0.0308\right)$ [53].

The initial feasible trajectory and velocity of UAVs are obtained as in [20]. UEs are randomly selected such that user selection constraint $\mathrm{C} 1$ is satisfied, and $p_{a}[n]=\frac{p_{t o t}}{4 T}, \hat{p}_{j}[n]=$ $\frac{p_{t o t}}{2 T}, \forall n$ are set fixed values such that constraints C3 and C5 are met initially. Note that one can readily obtain a feasible point of $\mathbf{P}$ by setting AP's transmit power to zero (or some non-zero but very small value to avoid numerical problems) and maximum AN's transmit powers to the average of the network power budget.

In Fig. 3, we plot mAEE versus iteration indices for different levels of covertness to demonstrate the convergence of the proposed iterative algorithm and verify the correctness of the analysis, i.e., the objective value of the optimization problem is non-decreasing over the iteration index. Note that the objective value obtained following the outer iterations are marked as blue diamonds on the curves. Although both the $\mathrm{CD}$ and TD schemes have fast convergence, the superiority of the JTCD compared to the other benchmarks is crystal clear; specifically, the proposed JTCD reaches the mAEE performance approximately quadruple as high as the $\mathrm{CD}$ counterpart at the end of the overall 6 iterations. We also observe from Fig. 3 that as $\varepsilon$ increases, i.e., the covertness constraint gets loosened, the adopted trajectory and resource allocations can be adjusted appropriately to acquire significantly higher mAEE for all schemes.

Fig. 4 illustrates how the minimum average covert throughput (mACT) and the average power consumption (APC) arising from UAVs propulsion vary over iteration numbers. Depending on the level of required covertness, we observe that the TD scheme can achieve comparably higher mACT performance than that of $\mathrm{CD}$, implying the significance of the trajectory design. Further, the benefits of the joint design shown in JTCD curves become more pronounced, particularly for a more strict covertness. Further, the mACT follows an overall decreasing trend as expected in order to improve the sum of ratios of energy efficiency performance metric.

In Figs. 5 and 6, we plot the 2D view of optimized trajectories of UIRS and UCJ using JTCD and TD schemes, respectively. Initial feasible trajectories are labeled as ite $=0$, which also belong to the $\mathrm{CD}$ scheme. The corresponding UIRS and UCJ's velocity are shown in Figs. $7 \mathrm{a}$ and $7 \mathrm{~b}$. We observe that over the iteration index, the path planning properly shapes with a relatively complicated form than the initial circular one to improve the system's mAEE performance. The trajectory variation between two consecutive iterations gets negligible as the algorithm approaches convergence.

Notice that UIRS should adjust its path flying towards the best location to obtain AP's data with low transmit power and then beamform towards the scheduled UE Bob at the given time slot while satisfying covertness requirement in terms of transmission detection failure of the strongest unscheduled user Willie. Therefore, for energy-efficient covert data transmission purposes, the UIRS's trajectory initially shrinks relatively towards Bob with the shortest distance, which can be verified in Fig. 8; nonetheless, adaptively gets adjusted when the UCJ's trajectory is updated. Further, in Fig. 6, the UCJ's designed path based on TD differs from the one obtained using the JTCD scheme due to a different set of the scheduled UEs over the time slots. However, in both scenarios, UCJ attempts to find a path with the highest detrimental effect on the Willies' detection rate while compensating such effect at Bob for the sake of mAEE improvement. Additionally, we can observe in Figs. $7 \mathrm{a}$ and $7 \mathrm{~b}$ that maintaining approximately fixed velocity or hovering at some particular locations for a while are not appropriate for energy-efficient design, which is fundamentally different from conventional designs focusing on solely covert data rate enhancement, e.g., [43]. Indeed, both UAVs must follow specific velocity adjustment patterns to reduce the mechanical power consumption while improving the covert throughput. Fig. 8 depicts the minimum error detection rate of each UE against the time slot for different algorithms. The absence of some curves at a particular time slot, e.g., UE \#1 curve between time slot $T=6 \mathrm{~s}$ and $T=11 \mathrm{~s}$ inclusive for the JTCD scheme, indicates the scheduled UE Bob. We observe that the minimum error detection rates stratify the covertness requirement for all scenarios, i.e., $\zeta_{m, k}^{\star} \geq 1-\varepsilon, \forall m, k$ with 

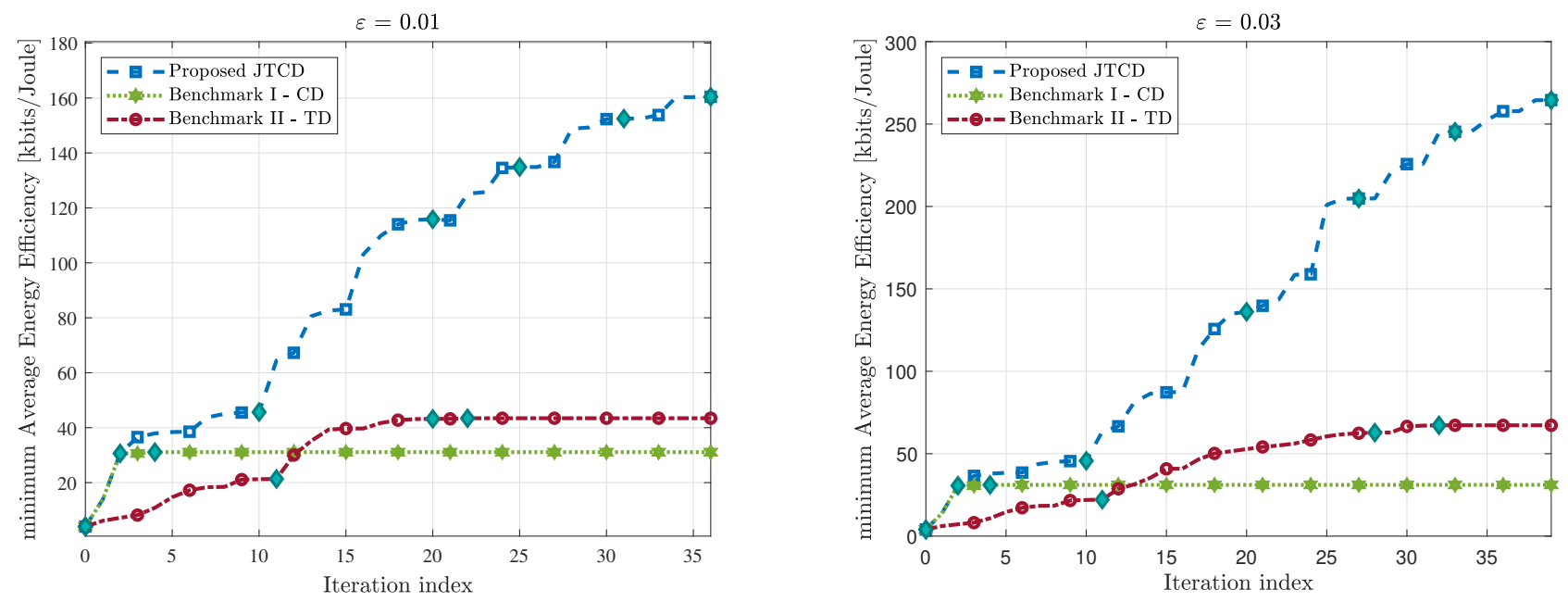

Fig. 3: mAEE vs Iteration index.

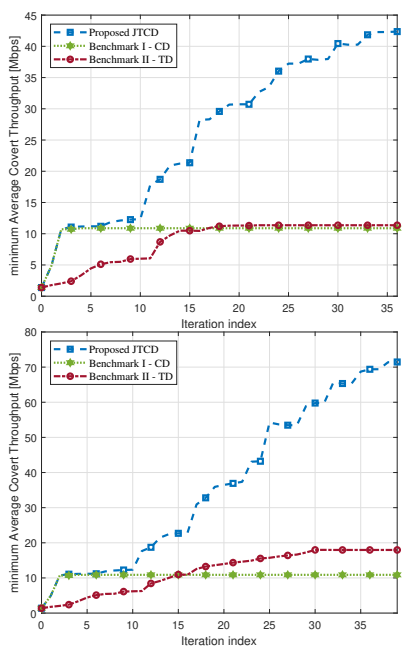

(a) $\varepsilon=0.01$.

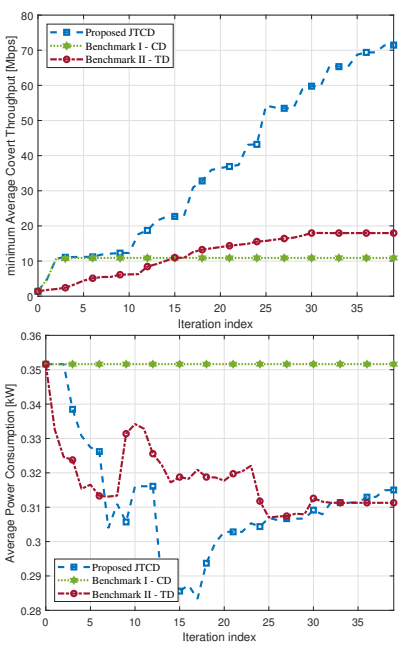

(b) $\varepsilon=0.03$.

Fig. 4: mACT and APC vs Iteration index.

$\varepsilon=0.01$ and the strongest detector Willie can achieve a minimum total error detection rate of no less than the required covertness. Further, with the fixed circular trajectory, we can see that mainly the closest users are scheduled for covert communication according to the CD scheme. However, when it comes to the JTCD scenario, based upon the level of AN transmission and the locations of UIRS and UCJ, a different set of UEs can be selected for energy-efficient covert transmission.

In Figs. 9 and 10, we examine the power allocation amongst AP's signal transmission and UCJ's AN transmission vs. time slot. Note that mAEE is an increasing function of AP's signal transmission power, while decreasing as AN transmission power increases. However, by proper resource and trajectory design, the level of maximum AN can be decreased without violating the covertness requirement. As an example, consider power allocation obtained according to Benchmark I with fixed circular UAVs' trajectories. Initially, UE \#5 is scheduled as Bob due to closeness to the UIRS according to Fig. 8 and so

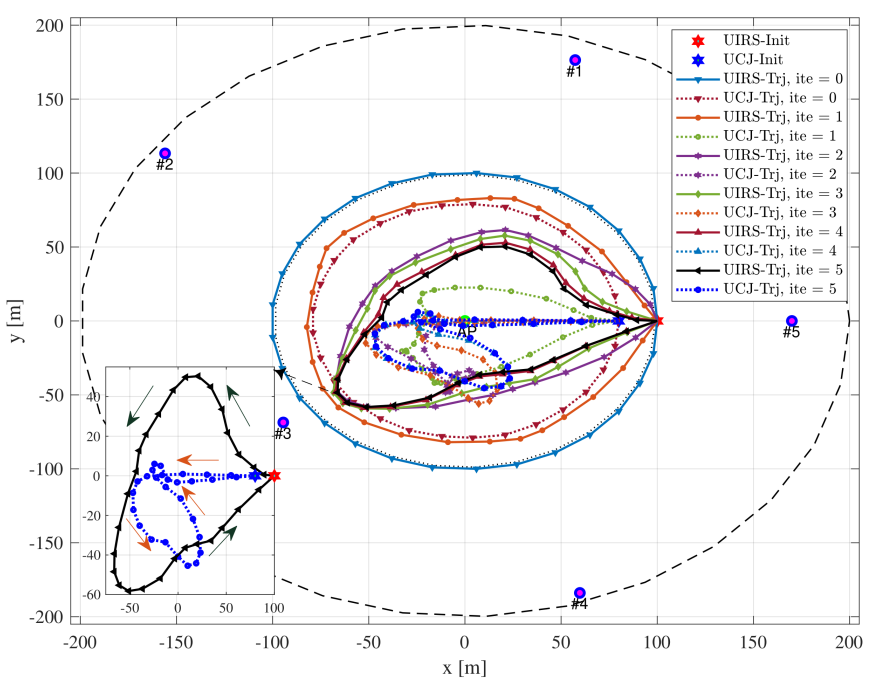

Fig. 5: UIRS and UCJ optimized trajectories according to the Proposed JTCD scheme.

AP transmits signals with its maximum instantaneous power; however, as the UIRS gets farther from Bob or closer to Willie with the strongest detector, i.e., UE \#1, AP's power drops while maximum AN transmission gets increased to satisfy the covertness requirement.

In the experiment depicted in Fig. 11, we compare how the mAEE performance varies with the increase of IRS elements for different scenarios. First, we note that the more the IRS elements, the higher the mAEE performance, owing to the fact that mAEE is an increasing function of $L$. However, by virtue of our worst-case design, an mAEE ceiling occurs at large $L$ due to the covertness constraint. Plus, such an increasing trend can be justified based on the fact that the IRS consists of a passive reflecting structure and does not cost any specific energy whilst improving the covert transmission rate. We see that the proposed JTCD scheme, Benchmark I - CD, and Benchmark II - TD, can increase mAEE by 


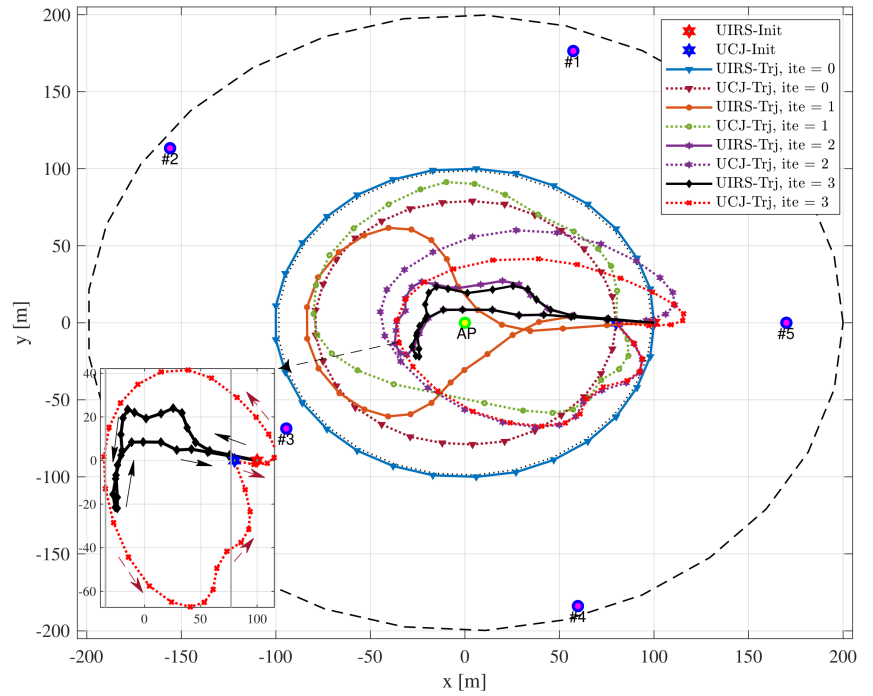

Fig. 6: UIRS and UCJ optimized trajectories according to Benchmark II.

approximately 22,9 , and 5 times, compared to the initial feasible point (Benchmark III - IFTR). When solely comparing Benchmark I and II schemes, Benchmark I - CD is the winner. This can be justified that although the trajectory design improves mAEE even with less number of IRS elements, resource allocation with a larger number of IRS elements dominates the final mAEE. Overall, we see that our proposed JTCD scheme outperforms other counterparts significantly for practical numbers of IRS elements.

Fig. 12 illustrates mAEE performance vs. carrier frequency, which also impacts the molecular absorption coefficient of $\mathrm{THz}$ links. As carrier frequency increases, more propagation loss and higher molecular absorption occur, bringing two impacts: one is on the reduced achievable covert rate and degraded mAEE performance, the other is on the diminished strength of the received signal at not only Bob, but also Willies. The former degrades the overall mAEE performance while the latter loosens the covertness constraint.

\section{$\mathrm{V}$ CONCLUSions}

This article addressed the energy-efficient design of a multi-UAV wireless communication system for covert data dissemination in the B5G UAV-IoT network operating at $\mathrm{THz}$ bands. Specifically, the UIRS is used for the passive covert data relaying from the AP to the scheduled UE, and the UCJ is employed for efficiently AN injection, degrading unscheduled UEs' detection performance. We showed analytically that properly employing a THz-operated UIRS-UCJ system can significantly improve communications reliability, capacity, coverage, and covertness, thanks to the dynamic nature of such a system. Then, the transmission detection performance from the perspective of unscheduled UEs as potential adversaries was explored, and the analytical closedform expressions were derived to evaluate covertness. Further, to improve the overall system performance, we developed a low-complex algorithm to iteratively solve a sequence of convex optimization problems for maximizing the mAEE

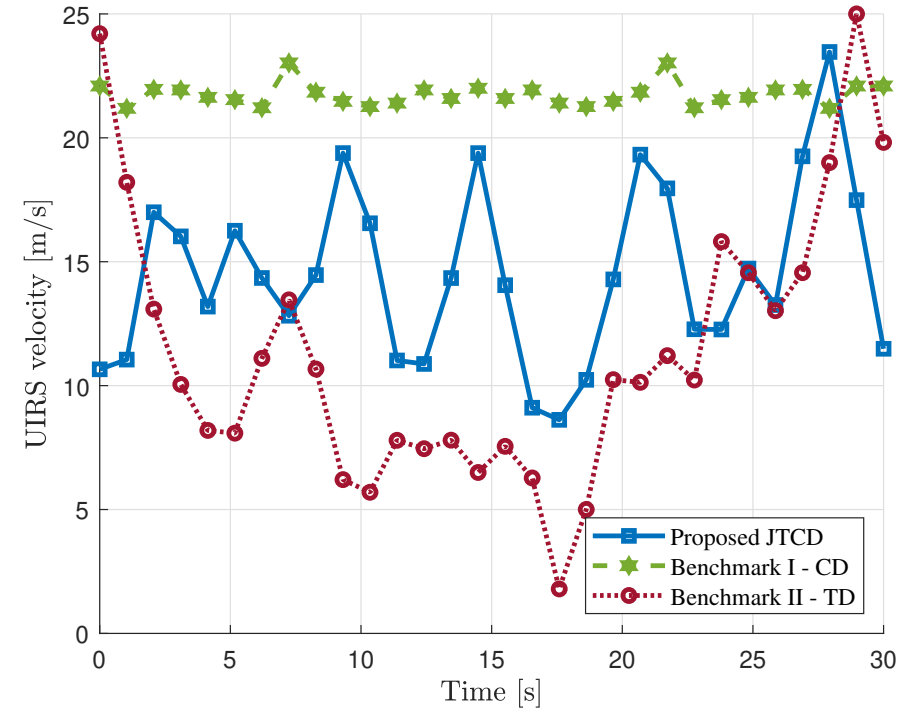

(a) UIRS optimized velocity.

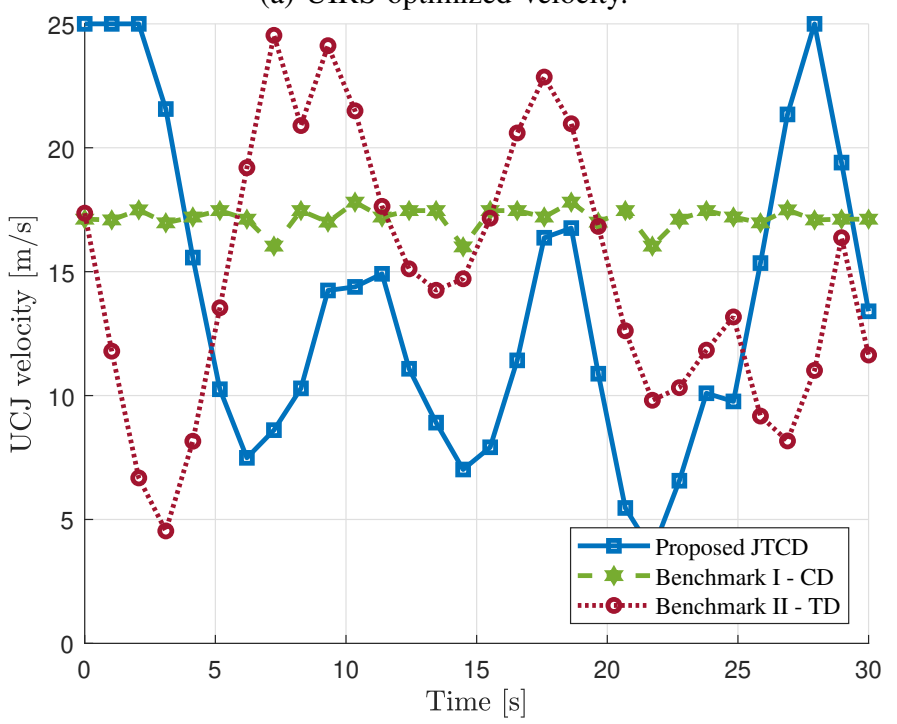

(b) UCJ optimized velocity.

Fig. 7: UIRS and UCJ's velocity according to different schemes with $T=30$ s and $\varepsilon=0.01$.

performance subject to the covertness requirement via the joint design of the user scheduling, network transmission power, IRS beamforming, and UAVs' trajectory planning. Our examination revealed significant outperformance of our proposed JTCD scheme compared to the other schemes in terms of both mAEE and mACT. Future work may focus on practical THz channel modeling and imperfect IRS phase shift and amplitude design for such a UAV-IoT system.

\section{APPENDIX A}

\section{JOINT DESIGN OF NETWORK POWER ALLOCATION AND} USER SCHEDULING 1

Here, we try to jointly optimize network transmission powers and user scheduling variables, i.e., $\left(\mathbf{P}_{a}, \hat{\mathbf{P}}_{j}, \boldsymbol{\alpha}\right)$. Therefore, by taking slack variables $\mathbf{w}=\left\{w_{k}[n], \forall n \in \mathcal{N}, k \in \mathcal{K}\right\}$, 


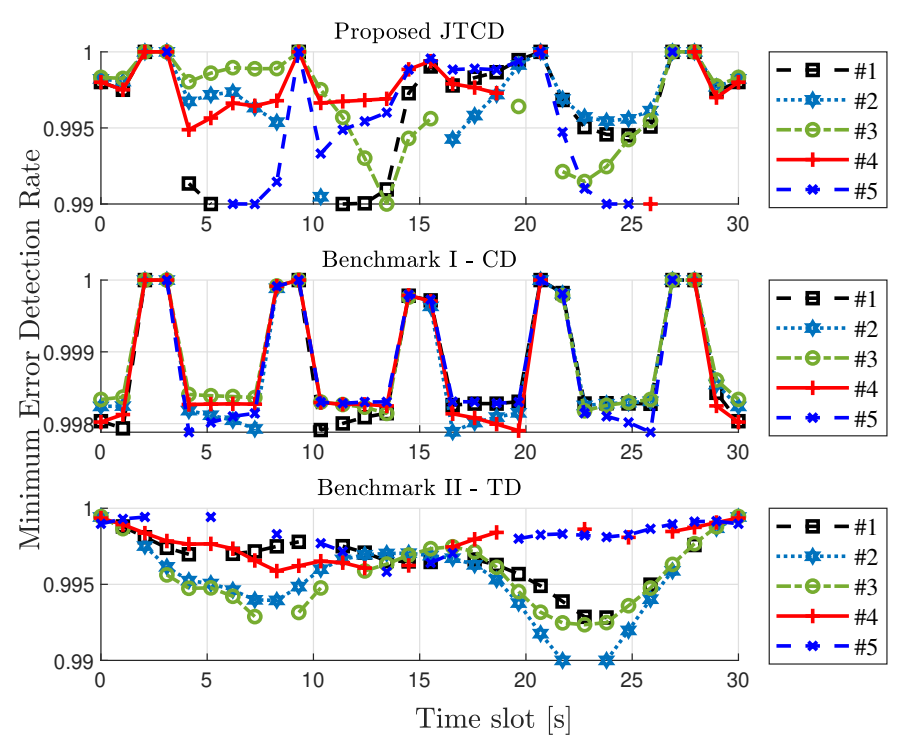

Fig. 8: $\zeta_{m, k}^{\star}$ and selected UEs according to different algorithms.

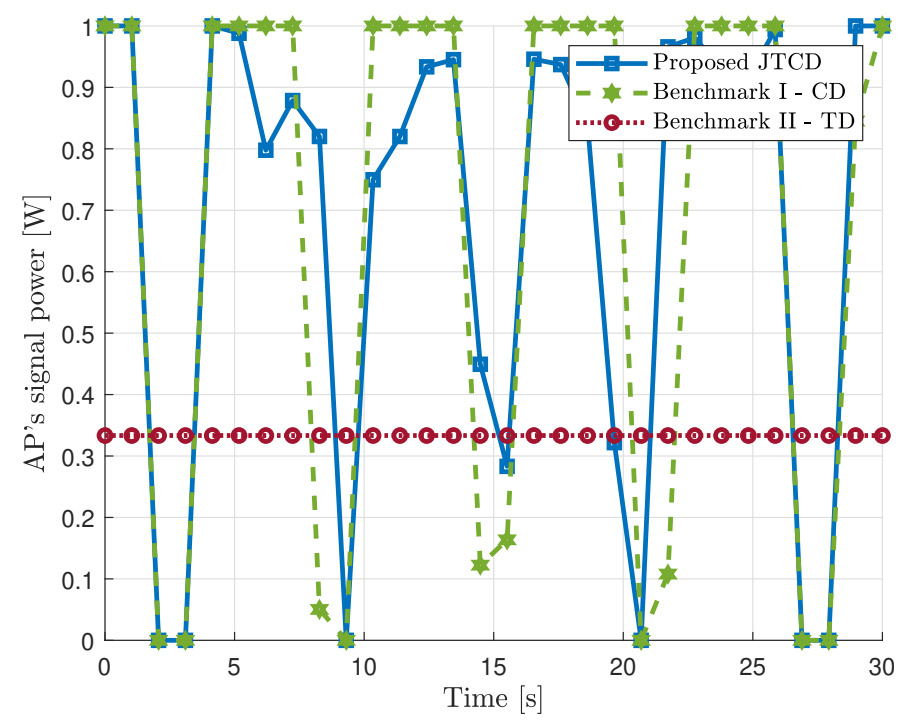

Fig. 9: AP's signal transmission power vs. time.

$\mathbf{v}=\left\{s_{k}[n], \forall n \in \mathcal{N}, k \in \mathcal{K}\right\}, \mathbf{r}=\{r[n], \forall n \in \mathcal{N}\}$, we can represent $(\mathrm{P})$ equivalently as

$$
\begin{aligned}
& (\widetilde{\mathrm{P} 3}): \underset{\psi, \boldsymbol{\alpha}, \mathbf{P}_{a}, \hat{\mathbf{P}}_{j}, \mathbf{w}, \mathbf{v}, \mathbf{r}}{\operatorname{maximize}} \psi \\
& \text { s.t. } \quad \frac{1}{N} \sum_{n=1}^{N} A_{n} \alpha_{k}[n] w_{k}[n] \geq \psi, \forall k \in \mathcal{K} \\
& \ln \left(1+\frac{B_{k, n} p_{a}[n]}{C_{k, n} \hat{p}_{j}[n]+1}\right) \geq w_{k}[n], \forall k \in \mathcal{K}, n \in \mathcal{N} \\
& \sum_{k=1}^{K} \alpha_{k}[n] s_{k}[n] \geq 1-\varepsilon, \forall n \in \mathcal{N} \\
& 1-s_{k}[n] \geq \max _{m \in \mathcal{W}}\left\{D_{n, k, m} \frac{p_{a}^{2}[n]}{r[n]}\right\}, \forall k \in \mathcal{K}, n \in \mathcal{N} \\
& r[n] \leq p_{a}[n] \hat{p}_{j}[n], \forall n \in \mathcal{N}
\end{aligned}
$$$$
\mathrm{C} 4 \text { \& } \mathrm{C} 6
$$

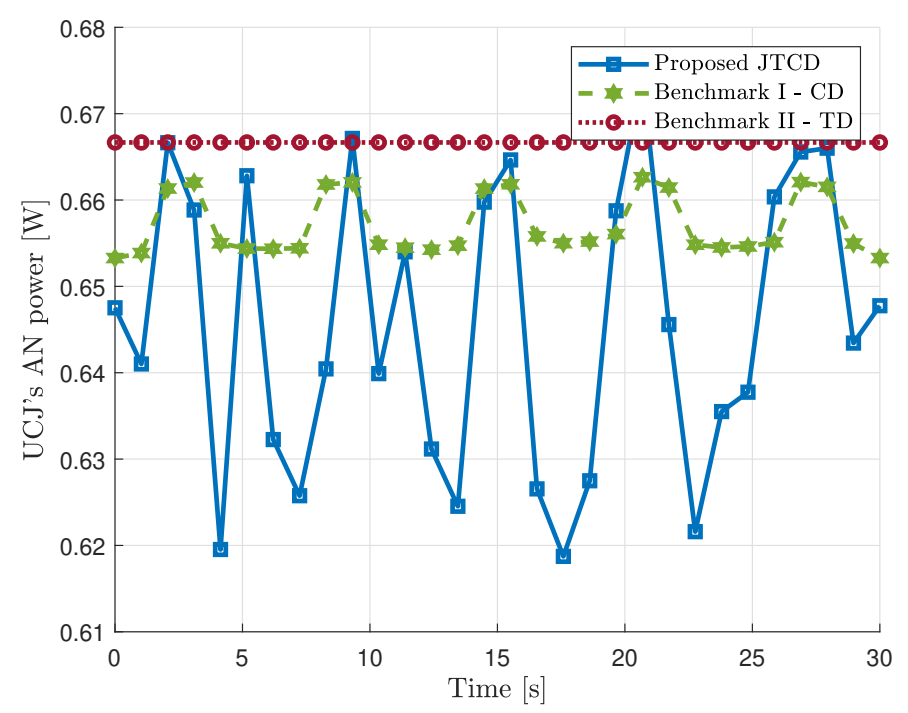

Fig. 10: Maximum UCJ's AN transmission power vs. time.

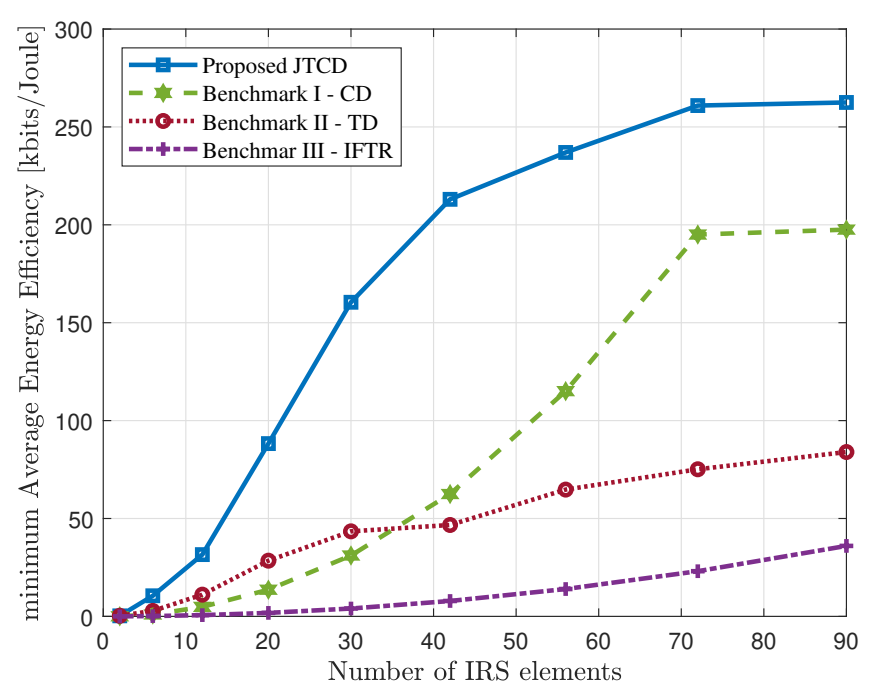

Fig. 11: Effect of number of IRS elements $L=L_{x} \times L_{y}$.

where $B_{k, n}, C_{k, n}, D_{n, k, m}$ are defined in (P2). Further, we have

$$
A_{n}=\frac{\mathrm{W}}{\ln (2)\left(P_{f, r}[n]+P_{f, j}[n]\right)}, \forall n \in \mathcal{N}
$$

We note that quadratic-over-linear is a convex function [49] and max of some convex functions is convex too, thus constraint (A.1d) is convex. Nonetheless, problem $(\widetilde{\mathrm{P} 3})$ is not convex due to nonconvex constraints (A.1a), (A.1b), (A.1c), (A.1e). To this end, we first mention lemma below to handle such challenging problem.

Lemma 4. Define the bi-variate function $f(x, y)=x y$ with $x \neq y$ which is a log-concave function. The global lowerbound and upper-bound of which at the given point $\left(x^{l o}, y^{l o}\right)$ can be obtained as

$$
\begin{aligned}
f(x, y) & \geq \frac{1}{4}\left(-\left(x^{l o}+y^{l o}\right)^{2}+2\left(x^{l o}+y^{l o}\right)(x+y)-(x-y)^{2}\right) \\
& \triangleq f_{c c v}^{l b}\left(x, y, x^{l o}, y^{l o}\right),
\end{aligned}
$$




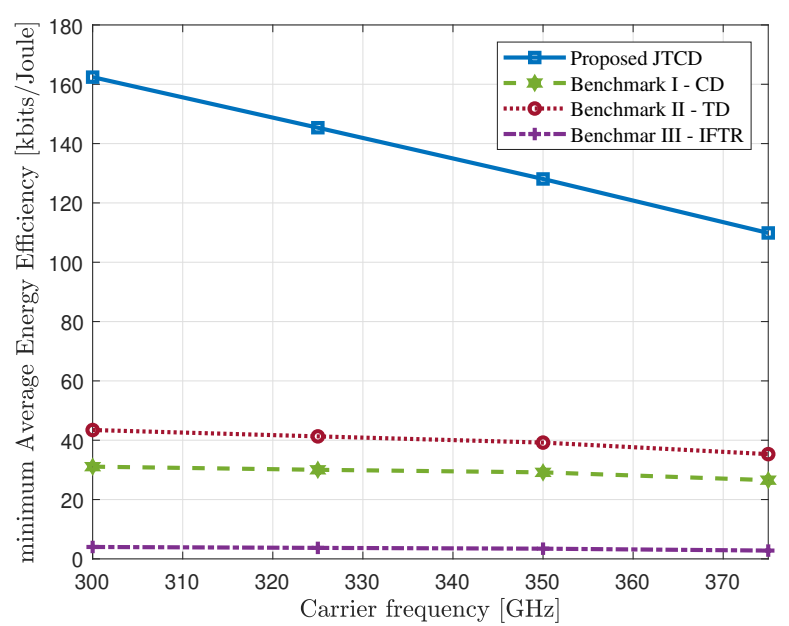

Fig. 12: Effect of carrier frequency on mAEE.

$$
\begin{aligned}
f(x, y) & \leq \frac{1}{4}\left((x+y)^{2}+\left(x^{l o}-y^{l o}\right)^{2}-2\left(x^{l o}-y^{l o}\right)(x-y)\right) \\
& \triangleq f_{c v x}^{u b}\left(x, y, x^{l o}, y^{l o}\right),
\end{aligned}
$$

Proof. Using the difference of quadratic reformulation, i.e., $x y=\frac{1}{4}\left[(x+y)^{2}-(x-y)^{2}\right]$ and properly employing the first order condition law [49] via applying restrictive approximation to the first or second quadratic terms, one can reach the aforementioned bounds.

Using Lemma 4 and the way we tackled binary use scheduling in $(\mathrm{P} 1.1)$, we can reformulate $(\widetilde{\mathrm{P} 3})$ as a convex optimization problem given by

$$
\begin{aligned}
& (\widetilde{\mathrm{P} 3.1}): \underset{\psi, \boldsymbol{\alpha}, \mathbf{P}_{a}, \hat{\mathbf{P}}_{j}, \mathbf{w}, \mathbf{v}, \mathbf{r}, \eta}{\operatorname{maximize}} \psi-\eta \mu \\
& \text { s.t. } \\
& \quad \frac{1}{N} \sum_{n=1}^{N} A_{n} f_{c c v}^{l b}\left(\alpha_{k}[n], w_{k}[n], \alpha_{k}^{l o}[n], w_{k}^{l o}[n]\right) \\
& \geq \psi, \forall k \in \mathcal{K} \\
& \ln \left(1+B_{n} p_{a}[n]+C_{n} \hat{p}_{j}[n]\right)-f_{l b}\left(\hat{p}_{j}[n], \hat{p}_{j}^{l o}[n]\right) \\
& \geq w_{k}[n], \quad \forall k \in \mathcal{K}, n \in \mathcal{N} \\
& \sum_{k=1}^{K} f_{c c v}^{l b}\left(\alpha_{k}[n], s_{k}[n], \alpha_{k}^{l o}[n], s_{k}^{l o}[n]\right) \geq 1-\varepsilon, \forall n \in \mathcal{N} \\
& f_{c c v}^{l b}\left(p_{a}[n], \hat{p}_{j}[n], p_{a}^{l o}[n], \hat{p}_{j}^{o}[n]\right) \geq r[n], \forall n \in \mathcal{N} \\
& \text { (A.1d) \& (A.1f) }
\end{aligned}
$$

where $\alpha_{k}^{l o}[n], w_{k}^{l o}[n], s_{k}^{l o}[n], \forall k, n$ are the given local points. Note that too many approximations are used to obtain the convex problem of (P3.1).

\section{APPENDIX B}

\section{PROOF OF LEMMA 1}

Given $\mathbf{A}$ be a complex square PSD matrix, we want to prove that $\mathbf{A}$ is Hermitian with nonnegative real eigenvalues. To this end, we first prove that every complex matrix can be written
$\mathbf{A}$ can be written as $\mathbf{A}=\mathbf{R}+j \mathbf{S}$ where $\mathbf{R}$ and $\mathbf{S}$ are two Hermitian matrices. We can commence by rewriting $\mathbf{A}$ as

$$
\mathbf{A}=\frac{1}{2}\left(\mathbf{A}+\mathbf{A}^{\dagger}\right)+\frac{1}{2}\left(\mathbf{A}-\mathbf{A}^{\dagger}\right)
$$

The first term of RHS, i.e. $\mathbf{R}=\frac{1}{2}\left(\mathbf{A}+\mathbf{A}^{\dagger}\right)$ is Hermitian, since $\mathbf{R}^{\dagger}=\mathbf{R}$ while the second term $\mathbf{T}=\frac{1}{2}\left(\mathbf{A}-\mathbf{A}^{\dagger}\right)$ is skew-Hermitian, i.e., $\mathbf{T}^{\dagger}=-\mathbf{T}$. Notice that if $\mathbf{T}$ is skew-Hermitian, then $\mathbf{S}=-j \mathbf{T}$ must be Hermitian, since $\mathbf{S}^{\dagger}=(-j \mathbf{T})^{\dagger}=-j \mathbf{T}=\mathbf{S}$. Therefore $\mathbf{A}=\mathbf{R}+j \mathbf{S}$ where $\mathbf{R}$ and $\mathbf{S}$ are both Hermitian. This representation is indeed unique. We use contradiction approach to prove the uniqueness of this decomposition. Suppose that $\mathbf{A}$ can also be written as $\mathbf{A}=\mathbf{R}^{\dagger}+j \mathbf{S}^{\dagger}$ with $\mathbf{R}^{\dagger} \neq \mathbf{R}$ and $\mathbf{S}^{\dagger} \neq \mathbf{S}$ being two Hermitian matrices. By subtracting the two forms of representations from each other we obtain $\mathbf{R}-\mathbf{R}^{\dagger}=-j\left(\mathbf{S}-\mathbf{S}^{\dagger}\right)$. Since $\mathbf{R}, \mathbf{R}^{\dagger}, \mathbf{S}, \mathbf{S}^{\dagger}$ are assumed to be Hermitian matrices, thus, $\mathbf{R}-\mathbf{R}^{\dagger}$ and $\mathbf{S}-\mathbf{S}^{\dagger}$ must also be Hermitian. However, here we have Hermitian matrix $\mathbf{R}-\mathbf{R}^{\dagger}$ being equal to a skewHermitian matrix $-j\left(\mathbf{S}-\mathbf{S}^{\dagger}\right)$ which is possible only if only possible if $\mathbf{R}-\mathbf{R}^{\dagger}=-j\left(\mathbf{S}-\mathbf{S}^{\dagger}\right)=0$ implying that $\mathbf{R}-\mathbf{R}^{\dagger}$ and $\mathbf{S}=\mathbf{S}^{\dagger}$. So, the Hermitian decomposition of a complex square matrix $\mathbf{A}=\mathbf{R}+j \mathbf{S}$ is unique. Assuming that $\mathbf{A} \in \mathbb{S}^{+}$ then by the definition $\mathbf{v}^{\dagger} \mathbf{A} \mathbf{v} \geq 0$ for an arbitrary vector $\mathbf{v}$. Letting $\mathbf{v}$ be the normalized eigenvector of matrix $\mathbf{S}$ with corresponding eigenvalue $\lambda_{v}$, then $\mathbf{v}^{\dagger} \mathbf{A} \mathbf{v}=\mathbf{v}^{\dagger} \mathbf{B} \mathbf{v}+j \lambda_{v}$. The LHS must be a non-negative real value according to the definition of PSD, thus, this is only possible if $\lambda_{v}=0$ resulting the fact that $\mathbf{A}=\mathbf{B}$ implying that $\mathbf{A}$ is Hermitian. Thus, any complex square PSD matrix is Hermitian with non-negative real eigenvalues. This completes the proof.

\section{REFERENCES}

[1] I. F. Akyildiz, A. Kak, and S. Nie, "6G and Beyond: The Future of Wireless Communications Systems," IEEE Access, vol. 8, pp. 133995 134 030, 2020.

[2] Y. Xu, Z. Liu, C. Huang, and C. Yuen, "Robust Resource Allocation Algorithm for Energy Harvesting-based D2D Communication Underlaying UAV-Assisted Networks," IEEE Internet Things J., vol. 4662, no. c, pp. $1-1,2021$.

[3] M. Tatar Mamaghani, A. Kuhestani, and H. Behroozi, "Can a multi-hop link relying on untrusted amplify-and-forward relays render security?" Wirel. Networks, vol. 27, no. 1, pp. 795-807, 2021.

[4] A. Li, W. Zhang, and S. Dou, "UAV-Enabled Secure Data Dissemination via Artificial Noise: Joint Trajectory and Communication Optimization," IEEE Access, vol. 8, pp. 102 348-102 356, 2020.

[5] W. Jiang, B. Han, M. A. Habibi, and H. D. Schotten, "The Road Towards 6G: A Comprehensive Survey,' IEEE Open J. Commun. Soc., vol. 2, pp. 334-366, 2021.

[6] Q. Wu, J. Xu, Y. Zeng, D. W. K. Ng, N. Al-Dhahir, R. Schober, and A. L. Swindlehurst, "A Comprehensive Overview on 5G-andBeyond Networks With UAVs: From Communications to Sensing and Intelligence," IEEE J. Sel. Areas Commun., vol. 39, no. 10, pp. 29122945 , oct 2021.

[7] "Commercial Drone Market Size." [Online]. Available: https://www.gminsights.com/toc/detail/ unmanned-aerial-vehicles-UAV-commercial-drone-market

[8] S. D. UNICEF, "How Drones Can Be Used to Combat COVID-19," pp. 1-4, 2020. [Online]. Available: https://www.unicef.org/supply/ documents/how-drones-can-be-used-combat-covid-19

[9] H. Shakhatreh, A. H. Sawalmeh, A. Al-Fuqaha, Z. Dou, E. Almaita, I. Khalil, N. S. Othman, A. Khreishah, and M. Guizani, "Unmanned Aerial Vehicles (UAVs): A Survey on Civil Applications and Key Research Challenges," IEEE Access, vol. 7, pp. 48 572-48 634, 2019. 
[10] S. Wan, J. Lu, P. Fan, and K. B. Letaief, "Toward Big Data Processing in IoT: Path Planning and Resource Management of UAV Base Stations in Mobile-Edge Computing System," IEEE Internet Things J., vol. 7, no. 7, pp. 5995-6009, jul 2020.

[11] M. Tatar Mamaghani and Y. Hong, "Improving PHY-Security of UAVEnabled Transmission With Wireless Energy Harvesting: Robust Trajectory Design and Communications Resource Allocation," IEEE Trans. Veh. Technol., vol. 69, no. 8, pp. 8586-8600, aug 2020.

[12] R. Ding, F. Gao, and X. S. Shen, "3D UAV Trajectory Design and Frequency Band Allocation for Energy-Efficient and Fair Communication: A Deep Reinforcement Learning Approach," IEEE Trans. Wirel. Commun., vol. 19, no. 12, pp. 7796-7809, 2020.

[13] M. Tatar Mamaghani and Y. Hong, "Joint Trajectory and Power Allocation Design for Secure Artificial Noise Aided UAV Communications," IEEE Trans. Veh. Technol., vol. 70, no. 3, pp. 2850-2855, mar 2021.

[14] H. Lei, D. Wang, K.-H. Park, I. S. Ansari, J. Jiang, G. Pan, and M.S. Alouini, "Safeguarding UAV IoT Communication Systems Against Randomly Located Eavesdroppers," IEEE Internet Things J., vol. 7, no. 2 , pp. $1230-1244$, feb 2020.

[15] Z. C. B. Zhang, Y. Zhang, Z. Niu, N. Kuang, W. Chen, L. Li, S. Li, and X. Ma, "A Survey on Terahertz Communications," China Commun., vol. 16, no. 2, pp. 1-35, 2019.

[16] H. Sarieddeen, N. Saeed, T. Y. Al-Naffouri, and M.-S. Alouini, "Next Generation Terahertz Communications: A Rendezvous of Sensing, Imaging, and Localization," IEEE Commun. Mag., vol. 58, no. 5, pp. 69-75, may 2020

[17] C. Chaccour, M. N. Soorki, W. Saad, M. Bennis, P. Popovski, and M. Debbah, "Seven Defining Features of Terahertz (THz) Wireless Systems: A Fellowship of Communication and Sensing," arXiv Prepr. arXiv2102.07668, feb 2021

[18] K. Tekbiyik, A. R. Ekti, G. K. Kurt, A. Gorcin, and H. Yanikomeroglu, "A Holistic Investigation of Terahertz Propagation and Channel Modeling toward Vertical Heterogeneous Networks," IEEE Commun. Mag., vol. 58, no. 11, pp. 14-20, nov 2020.

[19] L. Xu, M. Chen, M. Chen, Z. Yang, C. Chaccour, W. Saad, and C. S. Hong, "Joint Location, Bandwidth and Power Optimization for THzEnabled UAV Communications," IEEE Commun. Lett., vol. 25, no. 6, pp. 1984-1988, jun 2021

[20] M. Tatar Mamaghani and Y. Hong, "Terahertz-supported Untrusted UAVRelaying: Secrecy Energy Efficiency Maximization via Trajectory and Communication Co-design," arXiv Prepr. arXiv2107.09896, pp. 1-30, jul 2021.

[21] S. Basharat, S. Ali Hassan, H. Pervaiz, A. Mahmood, Z. Ding, and M. Gidlund, "Reconfigurable Intelligent Surfaces: Potentials, Applications, and Challenges for 6G Wireless Networks," IEEE Wirel. Commun., pp. $1-8$, jul 2021

[22] Q. Wu, S. Zhang, B. Zheng, C. You, and R. Zhang, "Intelligent Reflecting Surface-Aided Wireless Communications: A Tutorial," IEEE Trans. Commun., vol. 69, no. 5, pp. 3313-3351, may 2021.

[23] E. Bjornson, O. Ozdogan, and E. G. Larsson, "Reconfigurable Intelligent Surfaces: Three Myths and Two Critical Questions," IEEE Commun. Mag., vol. 58, no. 12, pp. 90-96, 2020.

[24] H. Lu, Y. Zeng, S. Jin, and R. Zhang, "Enabling Panoramic Full-Angle Reflection Via Aerial Intelligent Reflecting Surface," in 2020 IEEE Int. Conf. Commun. Work. (ICC Wkshps), jun 2020, pp. 1-6.

[25] M. Tatar Mamaghani, A. Kuhestani, and K.-K. Wong, "Secure TwoWay Transmission via Wireless-Powered Untrusted Relay and External Jammer," IEEE Trans. Veh. Technol., vol. 67, no. 9, pp. 8451-8465, 2018.

[26] M. Tatar Mamaghani and Y. Hong, "On the Performance of LowAltitude UAV-Enabled Secure AF Relaying With Cooperative Jamming and SWIPT," IEEE Access, vol. 7, pp. 153060-153 073, 2019.

[27] _ , "Intelligent Trajectory Design for Secure Full- Duplex MIMOUAV Relaying Against Active Eavesdroppers: A Model-Free Reinforcement Learning Approach," IEEE Access, vol. 9, pp. 4447-4465, 2021.

[28] Q. Wu and R. Zhang, "Intelligent Reflecting Surface Enhanced Wireless Network via Joint Active and Passive Beamforming," IEEE Trans. Wirel. Commun., vol. 18, no. 11, pp. 5394-5409, nov 2019.

[29] Y. Pan, K. Wang, C. Pan, H. Zhu, and J. Wang, "Sum Rate Maximization for Intelligent Reflecting Surface Assisted Terahertz Communications," arXiv Prepr. arXiv2008.12246, pp. 1-6, aug 2020.

[30] A. A. Deshpande, C. J. Vaca-Rubio, S. Mohebi, D. Salami, E. de Carvalho, P. Popovski, S. Sigg, M. Zorzi, and A. Zanella, "Energy-Efficient Design for RIS-assisted UAVcommunications in beyond-5G Networks," arXiv Prepr. arXiv2109.11933, sep 2021.
[31] X. Hong, P. Liu, F. Zhou, S. Guo, and Z. Chu, "Resource Allocation for Secure UAV-Assisted SWIPT Systems," IEEE Access, vol. 7, pp. 24 248-24 257, 2019.

[32] X. Sun, W. Yang, Y. Cai, R. Ma, and L. Tao, "Physical Layer Security in Millimeter Wave SWIPT UAV-Based Relay Networks," IEEE Access, vol. 7, pp. 35 851-35 862, 2019.

[33] C. Zhong, J. Yao, and J. Xu, "Secure UAV Communication With Cooperative Jamming and Trajectory Control," IEEE Commun. Lett., vol. 23 , no. 2, pp. 286-289, feb 2019.

[34] J. Tang, G. Chen, and J. P. Coon, "Secrecy performance analysis of wireless communications in the presence of UAV jammer and randomly located UAV eavesdroppers," IEEE Trans. Inf. Forensics Secur., vol. 14, no. 11 , pp. 3026-3041, 2019.

[35] G. Zhang, Q. Wu, M. Cui, and R. Zhang, "Securing UAV Communications via Joint Trajectory and Power Control," IEEE Trans. Wirel. Commun., vol. 18, no. 2, pp. 1376-1389, feb 2019.

[36] R. Soltani, D. Goeckel, D. Towsley, B. A. Bash, and S. Guha, "Covert Wireless Communication With Artificial Noise Generation," IEEE Trans. Wirel. Commun., vol. 17, no. 11, pp. 7252-7267, nov 2018.

[37] X. Lu, E. Hossain, T. Shafique, S. Feng, H. Jiang, and D. Niyato, "Intelligent Reflecting Surface Enabled Covert Communications in Wireless Networks," IEEE Netw., vol. 34, no. 5, pp. 148-155, sep 2020.

[38] M. Forouzesh, P. Azmi, A. Kuhestani, and P. L. Yeoh, "Joint Information-Theoretic Secrecy and Covert Communication in the Presence of an Untrusted User and Warden," IEEE Internet Things J., vol. 8, no. 9, pp. 7170-7181, may 2021.

[39] M. R. Bloch, "Covert Communication over Noisy Channels: A Resolvability Perspective," IEEE Trans. Inf. Theory, vol. 62, no. 5, pp. 2334 2354, 2016.

[40] B. A. Bash, D. Goeckel, and D. Towsley, "Limits of Reliable Communication with Low Probability of Detection on AWGN Channels," IEEE J. Sel. Areas Commun., vol. 31, no. 9, pp. 1921-1930, sep 2013.

[41] J. Kong, F. T. Dagefu, J. Choi, and P. Spasojevic, "Intelligent Reflecting Surface Assisted Covert Communication With Transmission Probability Optimization," IEEE Wirel. Commun. Lett., vol. 10, no. 8, pp. 18251829, aug 2021

[42] S. Yan, B. He, X. Zhou, Y. Cong, and A. L. Swindlehurst, "DelayIntolerant Covert Communications With Either Fixed or Random Transmit Power," IEEE Trans. Inf. Forensics Secur., vol. 14, no. 1, pp. 129140, jan 2019

[43] X. Zhou, S. Yan, F. Shu, R. Chen, and J. Li, "UAV-Enabled Covert Wireless Data Collection,” IEEE J. Sel. Areas Commun., vol. 39, no. 11, pp. 3348-3362, nov 2021.

[44] X. Jiang, X. Chen, J. Tang, N. Zhao, X. Y. Zhang, D. Niyato, and K.-K. Wong, "Covert Communication in UAV-Assisted Air-Ground Networks," IEEE Wirel. Commun., vol. 28, no. 4, pp. 190-197, aug 2021.

[45] Q. Zhang, W. Saad, and M. Bennis, "Reflections in the Sky: Millimeter wave communication with UAV-carried intelligent reflectors," 2019 IEEE Glob. Commun. Conf. GLOBECOM 2019 - Proc., 2019.

[46] Y. Zeng, J. Xu, and R. Zhang, "Energy minimization for wireless communication with rotary-wing UAV," IEEE Trans. Wirel. Commun., vol. 18, no. 4, pp. 2329-2345, 2019.

[47] A.-A. A. Boulogeorgos, E. N. Papasotiriou, and A. Alexiou, "A Distance and Bandwidth Dependent Adaptive Modulation Scheme for $\mathrm{THz}$ Communications," in 2018 IEEE 19th Int. Work. Signal Process. Adv. Wirel. Commun., vol. 2018-June, no. 1. IEEE, jun 2018, pp. 1-5.

[48] Y. Pan, K. Wang, C. Pan, H. Zhu, and J. Wang, "UAV-Assisted and Intelligent Reflecting Surfaces-Supported Terahertz Communications," IEEE Wirel. Commun. Lett., vol. 10, no. 6, pp. 1256-1260, jun 2021.

[49] S. Boyd and L. Vandenberghe, Convex Optimization. Cambridge university press, 2004.

[50] I. CVX Research, "CVX: Matlab Software for Disciplined Convex Programming, version 2.0," 2012.

[51] Z. Q. Luo, W. K. Ma, A. So, Y. Ye, and S. Zhang, "Semidefinite relaxation of quadratic optimization problems," IEEE Signal Process. Mag., vol. 27, no. 3, pp. 20-34, 2010.

[52] K. Shen and W. Yu, "Fractional Programming for Communication Systems-Part I: Power Control and Beamforming," IEEE Trans. Signal Process., vol. 66, no. 10, pp. 2616-2630, may 2018.

[53] H. Long, M. Chen, Z. Yang, B. Wang, Z. Li, X. Yun, and M. ShikhBahaei, "Reflections in the Sky: Joint Trajectory and Passive Beamforming Design for Secure UAV Networks with Reconfigurable Intelligent Surface," arXiv Prepr. arXiv2005.10559, may 2020. 


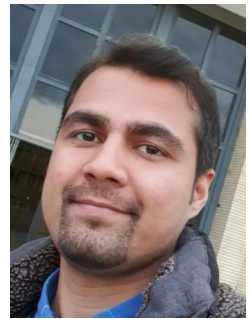

Milad Tatar Mamaghani (GS'20) was born in Tabriz, Iran, on May 12, 1994. He earned dual B.Sc. (Hons) degrees in electrical engineering fields Telecommunications and Control - from the Amirkabir University of Technology, Tehran, Iran, in 2016 and 2018, respectively. He is currently pursuing the $\mathrm{Ph} . \mathrm{D}$. degree with the Department of Electrical and Computer Systems Engineering, Monash University, Melbourne, Australia. He is the author of several papers published in prestigious journals, and has served as a volunteer reviewer of various reputable publication venues such as TWC, TIFS, TVT, TCOM, TCCN, TMC, ISJ, Access, WCL, etc. His research interests mainly focus on beyond $5 \mathrm{G}$ wireless communications and networking, physical-layer security, UAV communications, optimization, and artificial intelligence. He is a member of the IEEE Communications Society and the IEEE Signal Processing Society.

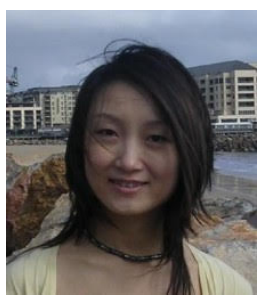

Yi Hong (S'00-M'05-SM'10) is currently an Associate Professor at the Department of Electrical and Computer Systems Eng., Monash University, Melbourne, Australia. She obtained her Ph.D. degree in Electrical Engineering and Telecommunications from the University of New South Wales (UNSW), Sydney, and received the NICTA-ACoRN Earlier Career Researcher Award at the 2007 Australian Communication Theory Workshop, Adelaide, Australia. She served on the Australian Research Council College of Experts (2018-2020). Yi Hong is currently an Associate Editor for IEEE Transactions on Green Communications and Networking, and was the Associate Editor for IEEE Wireless Communications Letters and Transactions on Emerging Telecommunications Technologies (ETT). She was the General Co-Chair of IEEE Information Theory Workshop 2014, Hobart; the Technical Program Committee Chair of Australian Communications Theory Workshop 2011, Melbourne; and the Publicity Chair at the IEEE Information Theory Workshop 2009, Sicily. She was a Technical Program Committee member for many IEEE leading conferences. Her research interests include communication theory, coding and information theory with applications to telecommunication engineering. 